\title{
Recruitment and organization of ESCRT-0 and ubiquitinated cargo via condensation
}

Sudeep Banjade, Lu Zhu, Jeffrey Jorgensen, Sho Suzuki and Scott D. Emr*

\author{
Weill Institute for Cell and Molecular Biology, Cornell University, Ithaca, NY, USA \\ Department of Molecular Biology and Genetics, Cornell University, Ithaca, NY, USA \\ *Correspondence to: Scott D. Emr (sde26@cornell.edu)
}

\begin{abstract}
The general mechanisms by which ESCRTs are specifically recruited to various membranes, and how ESCRT subunits are spatially organized remain central questions in cell biology. At the endosome and lysosomes, ubiquitination of membrane proteins triggers ESCRTmediated substrate recognition and degradation. Using the yeast lysosome/vacuole, we define the principles by which substrate engagement by ESCRTs occurs at this organelle. We find that multivalent interactions between ESCRT-0 and polyubiquitin is critical for substrate recognition at yeast vacuoles, with a lower-valency requirement for cargo engagement at endosomes. Direct recruitment of ESCRT-0 induces dynamic foci on the vacuole membrane, and forms fluid condensates in vitro with polyubiquitin. We propose that self-assembly of early ESCRTs induces condensation, an initial step in ESCRT-assembly/nucleation at membranes. This property can be tuned specifically at various organelles by modulating the number of binding interactions.
\end{abstract}

One-Sentence Summary: Condensation of multivalent ESCRT-0/polyubiquitin assemblies organizes cargo sorting reactions at lysosomes 
Main Text: ESCRT family members consist of a group of proteins involved in controlling diverse membrane-remodeling biochemical reactions, which include multivesicular body biogenesis, HIV budding, cytokinesis and membrane repair (1). Many of these occur at different membrane locations in the cell (endosome, lysosome, plasma membrane and the nucleus). Although the molecules that recruit ESCRTs are mostly known, the general principles of recruitment are less well understood. The mechanisms by which ESCRT reactions are spatially controlled remain unclear. In addition, while the structure of the individual subunits of the ESCRT complexes have been in most part solved (2-5), the mesoscale structural organization of early ESCRTs on the membrane is unclear.

At endosomes during multivesicular body biogenesis, ubiquitinated membrane proteins (cargo) recruit ESCRT-0, which then recruits the downstream proteins ESCRTs I, II and III(6). Therefore, ubiquitination serves as a signal to recruit ESCRTs to the endosomal membrane. The ESCRT-I complex has two ubiquitin binding motifs, ESCRT-II has one ubiquitin binding motif (7), and the complex of ESCRT-0 contains at least five ubiquitin binding motifs (one VHS and two UIM motifs in the ESCRT-0 protein Vps27, and one VHS and one UIM motif in the ESCRT-0 protein Hse1, (7)). Therefore, the ESCRT-0 complex is a critical component of the pathway that controls cargo binding. Furthermore, ESCRT-0 has been reported to form tetramers, oligomers and clusters $(8$, 9). In vivo cargo sorting occurs at hotspots $(10,11)$, demonstrating cargo concentration at specific sites, although the mechanism of this organization is not understood. The presence of multivalency for ubiquitin binding in ESCRT-0, and the knowledge of the existence of poly-ubiquitinated cargo as substrate, suggest higher order assembly of these complexes, as demonstrated for various multivalent interactions (12-14).

In this study, to understand the principles of ESCRT and cargo organization at membranes, we set out to delineate the properties of ESCRT's initial recruitment and assembly at two similar but separate organelles in yeast endosomes and vacuoles (yeast lysosomes). We recently described the mechanism of ESCRT-mediated lysosomal protein recognition and degradation via ubiquitination of lysosomal membrane proteins (15). While studying the requirements of vacuolar membrane protein recognition by ESCRTs, we find that polyubiquitination is critical for efficient ESCRT recognition at the vacuole. Higher valency of ubiquitin is more critical for cargo sorting at the 
vacuole than at endosomes. Polyubiquitinated proteins associate with ESCRT-0, leading to selfassembly of ESCRT-0 and cargo into dynamic condensates, which may provide a platform for nucleation of downstream ESCRT complexes.

As a model cargo, we used the vacuolar protease carboxy-peptidase-S (CPS), which is transported to the vacuole from the Golgi via the endosomal pathway(16). GFP-CPS is sorted into multivesicular bodies by the action of ESCRTs and is then trafficked to the vacuolar lumen for degradation (Fig. 1A). Therefore, GFP-CPS is localized in the vacuolar lumen (Fig. 1D, Fig. S1A). In ESCRT mutants (for example in the ESCRT-0 mutants vps $27 \Delta$ or $h s e 1 \Delta$ ), GFP-CPS gets stuck at aberrant endosomes (also called class-E compartments or "E-dots") (Fig. S1 B).

While Golgi-endosome-vacuole is one route for proteins to get to the vacuole lumen, another route to the vacuole is through the AP-3 pathway (Fig. 1A). When GFP-CPS is re-routed to the vacuole membrane by adding an AP-3 recognition site (hereafter called GFP-alp-CPS - "alp" for the "alp pathway"), the protein remains at the vacuole membrane (Fig. 1C-D, (16)). These two proteins (GFP-CPS and GFP-alp-CPS) therefore provide us with almost identical proteins that get to the vacuole via two independent vesicular compartments. This design allows us to probe the properties that ESCRTs utilize for the same protein to be recognized at two different physical locations in the cell.

Our previous study indicated that ESCRTs are able to recognize ubiquitinated membrane proteins at the vacuole membrane as well, in addition to endosomes (15). Considering that GFP-alp-CPS is stable at the vacuole membrane and does not get internalized into the lumen, we tested if conjugating CPS with ubiquitin induces ESCRT-mediated cargo internalization. We found that when a single ubiquitin is conjugated at the N-terminus of GFP-alp-CPS, the protein now gets internalized into the lumen, in a Vps27-dependent fashion (Fig. 1C-D, Fig. S1B).

Following this observation, we asked if a single ubiquitin is sufficient for complete internalization of GFP-alp-CPS on the vacuole membrane. Therefore, we used a lysine-less ubiquitin molecule (where all the seven lysines are mutated to arginines) to probe the effect of a polyubiquitin- 
deficient cargo. CPS normally gets ubiquitinated on K8 and K12 residues (17), which were mutated to Arg in this construct, and from hereon called Ub ${ }^{\mathrm{KR}}-\mathrm{GFP}-\mathrm{alp}-\mathrm{CPS}^{\mathrm{KR}}$ (Fig. 1C).

We found that the internalization of this polyubiquitin-deficient cargo is inhibited and a large fraction of it remains at the vacuole membrane (Fig. 1E). Compared with the normal ubiquitin containing lysines, the lysine-less protein does not form polyubiquitin chains (Fig. 1F). If the number of the lysine-less $\mathrm{Ub}$ molecules is increased making $2 \mathrm{X}-\mathrm{Ub}^{\mathrm{KR}}$ or $3 \mathrm{X}-\mathrm{Ub}^{\mathrm{KR}}$, internalization to the lumen is rescued (Fig. 1C-1D).

Vacuole-targeted membrane-protein cargoes are ubiquitinated through the action of E3 ligases such as Rsp5, Tul1 and Pib1 (18). Deletions of Rsp5-adaptors Ssh4 and Ear1, in addition to Tul1 and Pib1, had a severe defect in internalization of the vacuolar-membrane cargo Ub-GFP-alp-CPS (Fig. S2). These data provide evidence that a higher level of ubiquitination (or polyubiquitination) is required for the vacuolar membrane protein to be recognized and sorted by ESCRTs.

The data argue that the vacuolar membrane proteome is susceptible to regulation through the ESCRT pathway and that polyubiquitination of cargo is necessary for efficient sorting and degradation. To study the effect of the number of ubiquitin molecules on vacuolar protein cargo in a controlled fashion, we utilized a previously established rapamycin-dependent degradation system (15). In this system, the protein/cargo of interest is tagged with FKBP, and the yeast strain also contains an FRB molecule conjugated with ubiquitin. This system allowed us to control the number of ubiquitin molecules conjugated to the cargo of interest by following the kinetics of cargo sorting and degradation after addition of rapamycin, which triggers FRB-FKBP binding.

A 2X-FKBP (two FKBP molecules fused in tandem) in the presence of FRB-3XUb (three ubiquitin molecules in tandem) was effective in inducing ESCRT-mediated sorting of the vacuolar lysinetransporter Ypq1 (19). We modified the valency of ubiquitin bound to the cargo by mutating one of the FRB-binding sites in FKBP (called FKBP*), and by using single or triple ubiquitin fused to FRB (Figure 2A). In cargo sorting assays, we found a strong dependence on the number (valency) of ubiquitin fused to FRB (Fig. 2A-D, Fig. S3A-C). The same effect is also observed when we 
used an orthogonal cargo Vph1, with the number of effective ubiquitin molecules controlling the level of internalization into the vacuolar lumen (Fig. S3C).

To assess whether direct recruitment of ESCRTs to the vacuolar cargo can cause degradation of the cargo, we fused the ESCRT-0 protein Vps27 to FRB. This construct is fully functional and identical to the activity of the wild-type Vps27 (Fig. S4), as it can fully complement the defect of a vps $27 \Delta$ strain for two different cargo sorting reactions (Mup1-pHluorin and Can1 through a canavanine sensitivity assay, (20)).

Upon rapamycin-induced recruitment of Vps27 in this system, the modified cargo Ypq1 (fused to FKBP) internalized into the vacuolar lumen and degraded over time (Fig. 2E). Interestingly, we observed formation of distinct foci of the cargo at the vacuole surface upon adding rapamycin (Fig. 2E. Fig. S5). These foci are dynamic in nature - they move over time (Movie 1, Fig. S5), and occasionally fuse with one another. Some clusters disappear over time, as the cargo gets internalized into the lumen (Movie 1, Fig. S4C).

When a lower valency cargo construct is used instead, where one of the FKBP sites is mutated to abrogate FRB-Vps27 binding, upon rapamycin addition cargo clustering and sorting is inhibited (Fig. S6A). Importantly, this lower-valency cargo molecule is able to bind to FRB-Vps27 in a rapamycin-dependent fashion (Fig. S6B). Therefore, while ESCRT-0 is recruited, the valency of binding sites on the Ypq1 construct is critical to induce of ESCRT-mediated cargo sorting.

Our data therefore suggest that multivalent interactions between ESCRT-0 and cargo induce clustering, sorting and degradation. To understand the basis of cargo clustering induced by ESCRT-0, we purified the ESCRT-0 proteins Vps27 and Hse1, and reconstituted the assembly reaction in-vitro, using His6-tagged ubiquitin as the model cargo. We fused four ubiquitin genes in tandem with one another and labeled this molecule with Cy5. Upon assembling this model ubiquitin with ESCRT-0, micron sized condensates formed on supported lipid bilayers and in solution at low micromolar concentrations (Fig. 2F-H). The concentrations required for formation of condensates is lower in the nanomolar range on supported lipid bilayers and micromolar range 
in solution (Fig. 2F), implying robust formation of these assemblies. The structures also exhibit morphologies expected of liquid-like structures on membranes (Fig. 2F,(12)).

Inclusion of a single ubiquitin in the assay abrogated condensate formation, signifying the importance of ubiquitin valency in the self-assembly (Fig. 2G). A wide range of condensate sizes, down to nanometer scale are formed, as depicted by electron microscopy (3A). Rough edges of the condensates down to the nanometer level, and highly dense meshwork of proteins are reminiscent of network formation in such assemblies, as previously observed in other multivalent systems $(14,21)$.

These structures are dynamic since they exhibited molecular exchange ((Fig. 3B), fluorescence recovery after photobleaching). Solvent conditions are important for their formation, as condensation was favored by acidic $\mathrm{pH}$ (Fig. S7A,). These properties are hallmarks of biomolecular condensates, structures that exhibit dynamic rearrangement of molecules and rapid molecular exchange with the environment (13). These condensates did not form with only $4 \mathrm{XUb}$, or with $4 \mathrm{XUb}$ and Hse1 (Fig. S7B). Without Hse1, the condensates of $4 \mathrm{X} \mathrm{Ub}$ and Vps27 were smaller (Fig. S7B), as the valency of interaction is reduced. Condensate formation was possible but significantly inhibited with only Vps27 and Hse1, signifying weak self-association between the subunits of the ESCRT-0 complex (Fig. S7B), probably owing to the higher-order oligomerization and intrinsic disorder of the complex (Fig. S6C).

Mutations in the ubiquitin binding motifs have valency dependent effects on cargo sorting assays with the model endocytosis cargo Mup1 (Fig. 3D, 3F). Mutations in the ubiquitin-binding motifs of Vps27 (Vps27 vhs uim uim) also inhibited condensate formation in-vitro (Fig. 3E).

Our data therefore imply that the role of multivalent interactions during cargo sorting is to cluster cargo into condensates. Higher valency interactions through polyubiquitin and ESCRT-0 is also critical for cargo sorting reactions at the vacuolar membrane for several cargoes (GFP-alp-CPS, Ypq1, Vph1) and also for endosomal cargo (Mup1). Interestingly, the internalization of the lysineless GFP-CPS molecule, (Ub ${ }^{\mathrm{KR}}$-GFP-CPS ${ }^{\mathrm{KR}}$ : note without the alp signal), that traffics through the endosome, localizes to the lumen of the vacuole (Fig. 4A), in contrast to the vacuolar cargo (Fig. 
1). Therefore, the cargo recognition and internalization of this polyubiquitin-deficient membrane protein is more severely defective at the vacuolar membrane compared to the endosomal membrane. These effects are observed also when we follow the kinetics of degradation of the vacuole-targeted cargo compared with that of the endosome-targeted cargo. We used a galactose inducible expression system, following the stability of the protein over time upon translation inhibition with cycloheximide (Figure 4A). Although the proteins reach the endosome/vacuole membrane (Figure 4C), the kinetics of degradation of the vacuole targeted protein is much slower than that of the endosome-targeted protein (Fig. 4A-B). The rate of degradation can be enhanced with a $3 \mathrm{XUb}^{\mathrm{KR}}$ conjugated molecule (Fig. S8). These data imply that lower valency interactions are more efficient for cargo sorting at endosomes than at vacuolar membrane.

Endosomes are sites of high density of ESCRTs (22). We reasoned that due to the higher density of ESCRTs at the endosomes, there is a lower requirement for multivalent interactions to recruit and condense ESCRTs at this organelle, as opposed to the vacuolar membrane. One of the lipidspecies that recruit ESCRTs to the endosomal membrane is phosphatidyl-inositol-3P (PI3P). We hypothesized that an increase in PI3P density could in turn increase the density of ESCRTs, thereby reducing the requirement of higher valency interactions at the vacuolar membrane. To test this prediction, we used the PI3P phosphatase mutants $y m r 1 \Delta$ and $s j l 3 \Delta$, which have previously been shown to increase the level of PI3P on the vacuole membrane (23). In the single mutants ymr1 $\Delta$ and $s j l 3 \Delta$, the single-ubiquitin conjugated vacuolar GFP-CPS ( $\mathrm{Ub}^{\mathrm{KR}}$-GFP-alp-CPS $\left.{ }^{\mathrm{KR}}\right)$ remains mostly on the vacuole membrane (Fig. S9B). However, in the double mutant $y m r 1 \Delta$ sjl3 $\Delta$, the molecule is enriched in the vacuole lumen (Fig. 4D). Therefore, the defective sorting of the substrate can be rescued by the local increase in density of PI3P at the vacuolar membrane.

Our data provide evidence for two distinct mechanisms of recruitment and organization of ESCRT complexes at two separate membrane locations: the endosomes and the vacuole (Fig. 4D). The general principles behind function of ESCRTs at membranes were proposed to include recruitment to membranes, "self-assembly", and ESCRT-III polymerization (24). Our data suggest that one of the functions of the ESCRT-0 complex, by virtue of having multiple binding sites for ubiquitin, is to facilitate cargo clustering at the membrane through biomolecular condensation. ESCRT-0 by itself has a weaker ability to self-associate and undergo condensation. When 
substrates are polyubiquitinated, the multivalency of ESCRT-0 enhances formation of higherorder species of the ESCRT-0/cargo complexes. Increasing the density of PI3P increases the local concentration of ESCRT-0/ubiquitin on the membrane, thereby lowering the threshold for selfassociation (Fig. 4D, S10).

Condensing cargo via early ESCRTs could provide multiple advantages in the cargo-sorting process. The physical concentration of cargo could amplify sorting of cargo, increasing the specificity for cargo recognition. Concentrating downstream ESCRT complexes at a particular location and increasing the dwell time on the membrane could also provide a platform for nucleation of ESCRT-III polymerization, as observed for actin assembly pathways through upstream condensing nucleators (25). The rapid dynamics of the ESCRT-ubiquitin complexes also could allow for facile dissociation of the ESCRTs from cargo. Furthermore, condensation of ubiquitinated cargo could feed-back to the ubiquitin-ligase machinery, allowing for enhanced ubiquitination of the cargo, further providing specificity for cargo recognition.

At locations in the cell where ESCRT-0 is not involved, there may exist other mechanisms of selfassembly - in the case of HIV budding, the HIV Gag protein is known to form higher-order assemblies, which recruit downstream ESCRTs (26). Clustering and condensation of ESCRTrecruiters, therefore, could be a general property of ESCRT-related systems.

Our data provide clues for how clustering of signaling molecules can be regulated at various locations in the cell. By changing the number of binding sites for a complex on the membrane, cells may be able to quickly adjust local concentrations of enzymes and signaling adaptors. 2D surfaces and their charge properties may also play an important role in the nucleation of condensates (27) and also promote membrane repair and remodeling $(28,29)$. The composition of the membrane therefore should play a critical role in controlling formation and function of membrane-associated biomolecular condensates. 


\section{References:}

1. M. Vietri, M. Radulovic, H. Stenmark, The many functions of ESCRTs. Nat. Rev. Mol. Cell Biol. 21, 25-42 (2020).

2. M. S. Kostelansky, C. Schluter, Y. Y. C. Tam, S. Lee, R. Ghirlando, B. Beach, E. Conibear, J. H. Hurley, Molecular architecture and functional model of the complete yeast ESCRT-I heterotetramer. Cell. 129, 485-498 (2007).

3. J. McCullough, A. K. Clippinger, N. Talledge, M. L. Skowyra, M. G. Saunders, T. V. Naismith, L. A. Colf, P. Afonine, C. Arthur, W. I. Sundquist, P. I. Hanson, A. Frost, Structure and membrane remodeling activity of ESCRT-III helical polymers. Science. 350, 1548-1551 (2015).

4. S. Tang, W. M. Henne, P. P. Borbat, N. J. Buchkovich, J. H. Freed, Y. Mao, J. C. Fromme, S. D. Emr, Structural basis for activation, assembly and membrane binding of ESCRT-III Snf7 filaments. Elife. 4 (2015), doi:10.7554/eLife.12548.

5. T. G. Flower, Y. Takahashi, A. Hudait, K. Rose, N. Tjahjono, A. J. Pak, A. L. Yokom, X. Liang, H.-G. Wang, F. Bouamr, G. A. Voth, J. H. Hurley, A helical assembly of human ESCRT-I scaffolds reverse-topology membrane scission. Nat Struct Mol Biol. 27, 570-580 (2020).

6. D. J. Katzmann, C. J. Stefan, M. Babst, S. D. Emr, Vps27 recruits ESCRT machinery to endosomes during MVB sorting. J Cell Biol. 162, 413-423 (2003).

7. J. H. Hurley, S. D. Emr, The ESCRT complexes: structure and mechanism of a membranetrafficking network. Annu Rev Biophys Biomol Struct. 35, 277-298 (2006).

8. T. Wollert, J. H. Hurley, Molecular mechanism of multivesicular body biogenesis by ESCRT complexes. Nature. 464, 864-869 (2010).

9. J. R. Mayers, I. Fyfe, A. L. Schuh, E. R. Chapman, J. M. Edwardson, A. Audhya, ESCRT-0 assembles as a heterotetrameric complex on membranes and binds multiple ubiquitinylated cargoes simultaneously. J Biol Chem. 286, 9636-9645 (2011).

10. E. B. Frankel, A. Audhya, ESCRT-dependent cargo sorting at multivesicular endosomes. Semin Cell Dev Biol. 74, 4-10 (2018).

11. M. A. Y. Adell, S. M. Migliano, S. Upadhyayula, Y. S. Bykov, S. Sprenger, M. Pakdel, G. F. Vogel, G. Jih, W. Skillern, R. Behrouzi, M. Babst, O. Schmidt, M. W. Hess, J. A. Briggs, T. Kirchhausen, D. Teis, Recruitment dynamics of ESCRT-III and Vps4 to endosomes and implications for reverse membrane budding. Elife. 6 (2017), doi:10.7554/eLife.31652.

12. S. Banjade, M. K. Rosen, Phase transitions of multivalent proteins can promote clustering of membrane receptors. Elife. 3 (2014), doi:10.7554/eLife.04123. 
13. S. F. Banani, H. O. Lee, A. A. Hyman, M. K. Rosen, Biomolecular condensates: organizers of cellular biochemistry. Nat Rev Mol Cell Biol. 18, 285-298 (2017).

14. P. Li, S. Banjade, H.-C. Cheng, S. Kim, B. Chen, L. Guo, M. Llaguno, J. V. Hollingsworth, D. S. King, S. F. Banani, P. S. Russo, Q.-X. Jiang, B. T. Nixon, M. K. Rosen, Phase transitions in the assembly of multivalent signalling proteins. Nature. 483, 336-340 (2012).

15. L. Zhu, J. R. Jorgensen, M. Li, Y.-S. Chuang, S. D. Emr, ESCRTs function directly on the lysosome membrane to downregulate ubiquitinated lysosomal membrane proteins. Elife. 6 (2017), doi:10.7554/eLife.26403.

16. G. Odorizzi, M. Babst, S. D. Emr, Fab1p PtdIns(3)P 5-kinase function essential for protein sorting in the multivesicular body. Cell. 95, 847-858 (1998).

17. D. J. Katzmann, M. Babst, S. D. Emr, Ubiquitin-dependent sorting into the multivesicular body pathway requires the function of a conserved endosomal protein sorting complex, ESCRT-I. Cell. 106, 145-155 (2001).

18. X. Yang, W. Zhang, X. Wen, P. J. Bulinski, D. A. Chomchai, F. M. Arines, Y.-Y. Liu, S. Sprenger, D. Teis, D. J. Klionsky, M. Li, TORC1 regulates vacuole membrane composition through ubiquitin- and ESCRT-dependent microautophagy. J Cell Biol. 219, e201902127 (2020).

19. M. Li, Y. Rong, Y.-S. Chuang, D. Peng, S. D. Emr, Ubiquitin-dependent lysosomal membrane protein sorting and degradation. Mol Cell. 57, 467-478 (2015).

20. S. Banjade, S. Tang, S. D. Emr, Genetic and Biochemical Analyses of Yeast ESCRT. Methods Mol. Biol. 1998, 105-116 (2019).

21. T. M. Franzmann, M. Jahnel, A. Pozniakovsky, J. Mahamid, A. S. Holehouse, E. Nüske, D. Richter, W. Baumeister, S. W. Grill, R. V. Pappu, A. A. Hyman, S. Alberti, Phase separation of a yeast prion protein promotes cellular fitness. Science. 359 (2018), doi:10.1126/science.aao5654.

22. D. Teis, S. Saksena, B. L. Judson, S. D. Emr, ESCRT-II coordinates the assembly of ESCRT-III filaments for cargo sorting and multivesicular body vesicle formation. EMBO J. 29, 871-883 (2010).

23. W. R. Parrish, C. J. Stefan, S. D. Emr, Essential role for the myotubularin-related phosphatase Ymrlp and the synaptojanin-like phosphatases Sj12p and Sj13p in regulation of phosphatidylinositol 3-phosphate in yeast. Mol Biol Cell. 15, 3567-3579 (2004).

24. J. Votteler, C. Ogohara, S. Yi, Y. Hsia, U. Nattermann, D. M. Belnap, N. P. King, W. I. Sundquist, Designed proteins induce the formation of nanocage-containing extracellular vesicles. Nature. 540, 292-295 (2016).

25. L. B. Case, X. Zhang, J. A. Ditlev, M. K. Rosen, Stoichiometry controls activity of phaseseparated clusters of actin signaling proteins. Science. 363, 1093-1097 (2019). 
26. L.-A. Carlson, J. A. G. Briggs, B. Glass, J. D. Riches, M. N. Simon, M. C. Johnson, B. Müller, K. Grünewald, H.-G. Kräusslich, Three-Dimensional Analysis of Budding Sites and Released Virus Suggests a Revised Model for HIV-1 Morphogenesis. Cell Host \& Microbe. 4, 592-599 (2008).

27. W. T. Snead, A. S. Gladfelter, The Control Centers of Biomolecular Phase Separation: How Membrane Surfaces, PTMs, and Active Processes Regulate Condensation. Mol Cell. 76, 295-305 (2019).

28. A. von Appen, D. LaJoie, I. E. Johnson, M. J. Trnka, S. M. Pick, A. L. Burlingame, K. S. Ullman, A. Frost, LEM2 phase separation promotes ESCRT-mediated nuclear envelope reformation. Nature. 582, 115-118 (2020).

29. F. Yuan, H. Alimohamadi, B. Bakka, A. N. Trementozzi, K. J. Day, N. L. Fawzi, P. Rangamani, J. C. Stachowiak, Membrane bending by protein phase separation. Proc Natl Acad Sci U S A. 118, e2017435118 (2021).

\section{Acknowledgments}

We thank all the past and current members of the Emr lab for valuable discussions. Sudeep Banjade was an HHMI fellow of the Damon Runyon Cancer Research Foundation. This work was also supported by a Cornell University Grant to Scott D Emr.

\section{Funding}

Damon Runyon Cancer Research Fellowship (through HHMI): DRG-2273-16

Cornell University Grant CU3704 to Scott D. Emr

\section{Author Contributions}

Conceptualization: SB

Methodology: SB, LZ, JJ, SS

Investigation: $\mathrm{SB}$

Funding acquisition: SB, SDE

Supervision: SDE

Writing - original draft: SB

Writing - review \& editing: SB, SS, SDE 


\section{Figure 1}
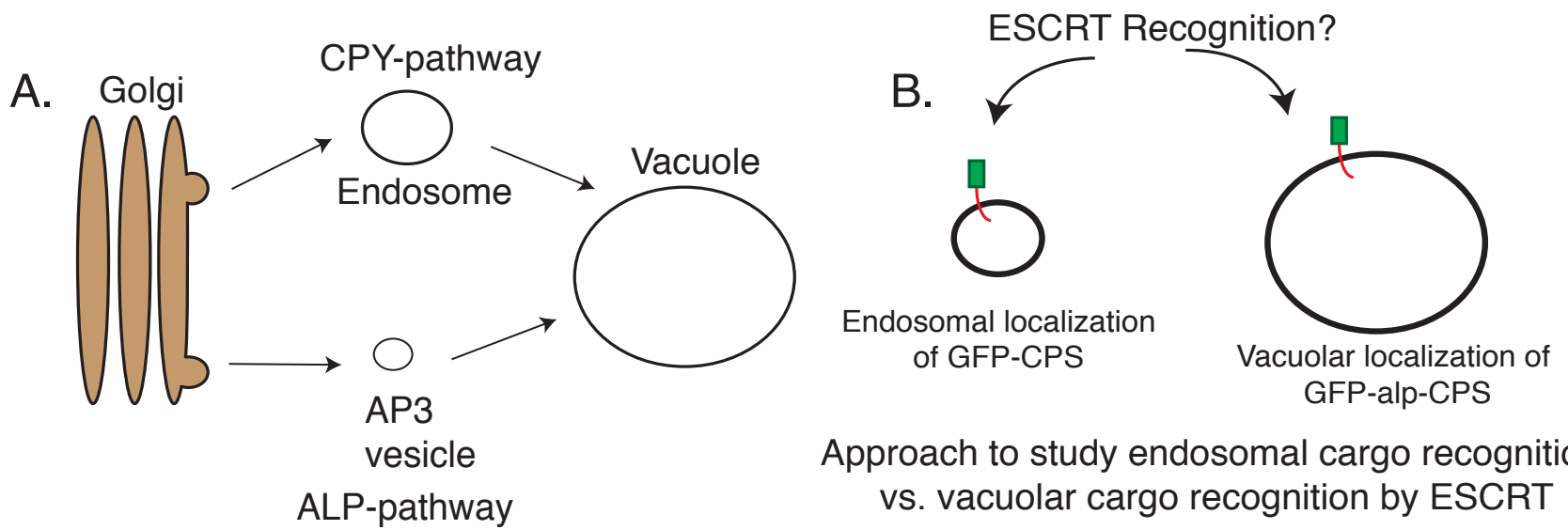

C.

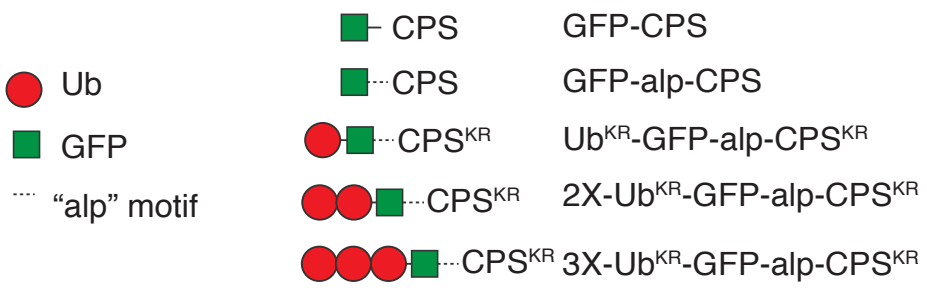

D.
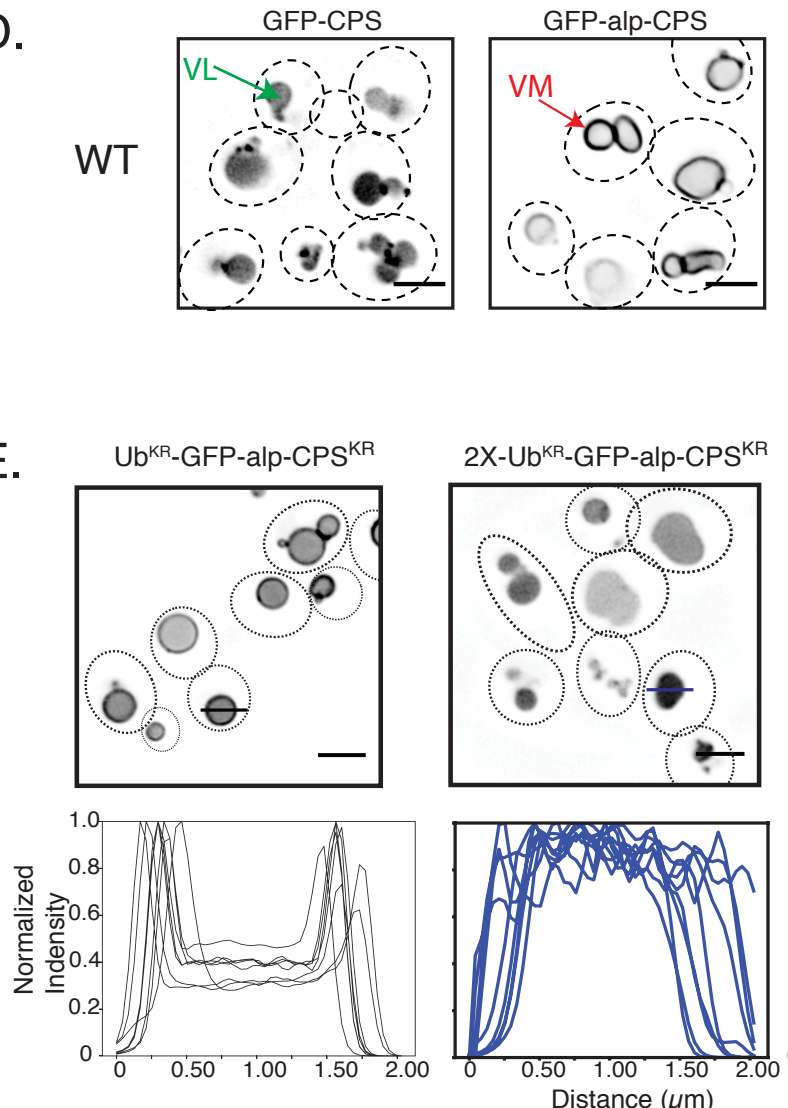

Ub-GFP-alp-CPS

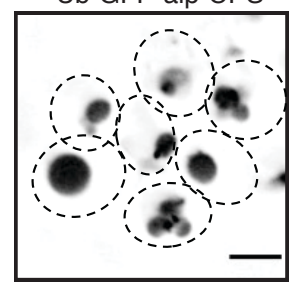

VL: vacuole lumen vacuole membrane

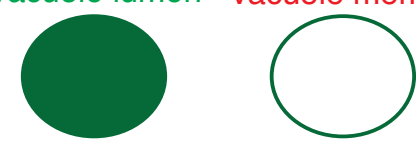

3X-Ub ${ }^{\mathrm{KR}}$-GFP-alp-CPS ${ }^{\mathrm{KR}}$
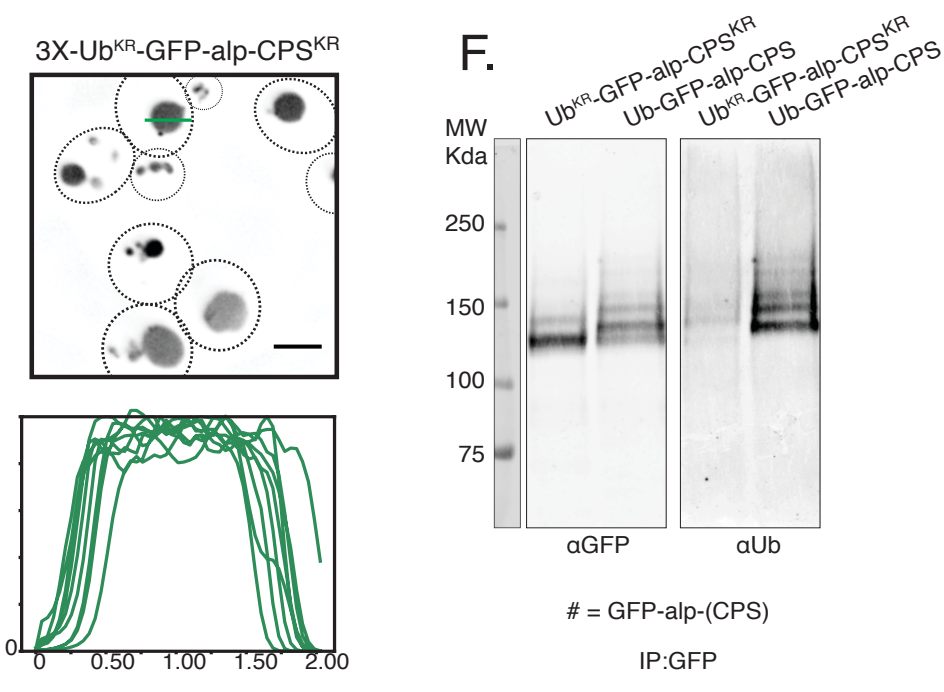
Fig. 1. Polyubiquitination is required for efficient ESCRT-dependent cargo sorting at the vacuole. A) Model of carboxy peptidase S (CPS) and ALP trafficking to the vacuole. CPS traffics through the "CPY-pathway"/endosome where MVBs are formed by ESCRTs. The ALP-pathway forms separate vesicles that traffics proteins like the alkaline phosphatase to the vacuole. B) Model for the localization of GFP-CPS and GFP-alp-CPS at two sites where ESCRTs can function, providing a tool to study the same cargo at two membrane locations in the cell. C) Different CPS constructs used in this experiment. D)Localization of the CPS constructs as listed on top of the figures in a WT strain. The dotted lines represent outlines of yeast cells as imaged through the DIC channel. For simplicity the DIC channel is not shown but some images with the DIC channel are shown in the supplements (Fig S1). Note that VL denotes vacuolar lumen, VM denotes vacuole membrane. Right: A model of different variants of GFP-CPS localizing in the vacuolar lumen(VL) or vacuolar membrane (VM). Scale bars are $2 \mu \mathrm{m}$ long. E) Localization of the GFP-CPS constructs as denoted in the figure. $\mathrm{UB}^{\mathrm{KR}}$ has all the lysines mutated to Arg. Intensity profiles on the bottom represent line scan across the vacuoles. Representative lines are shown across three vacuoles (black, blue and green lines) in the microscopy images. Scale bars are $2 \mu \mathrm{m}$ long. F) Immunoblots of $U^{\mathrm{KR}}{ }_{-G F P-a l p-C P S}{ }^{\mathrm{KR}}$ (lysine-less substrate) and Ub-GFP-alp-CPS after performing immunoprecipitation with anti-GFP. These experiments were performed in a doa $4 \Delta$ pep $4 \Delta$ prb1 $\Delta$ background strain. 
bioRxiv preprint doi: https://doi.org/10.1101/2021.08.23.457352; this version posted August 23, 2021. The copyright holder for this preprint (which was not certified by peer review) is the author/funder, who has granted bioRxiv a license to display the preprint in perpetuity. It is made available under aCC-BY-NC-ND 4.0 International license.

Figure 2

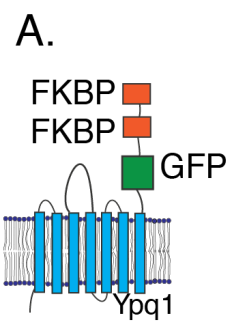

B.

Ypq1-FKBP-FKPB-GFP

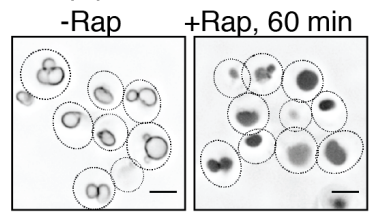

C.

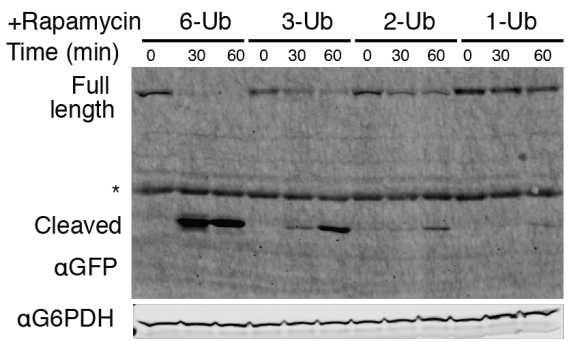

+Rapamycin

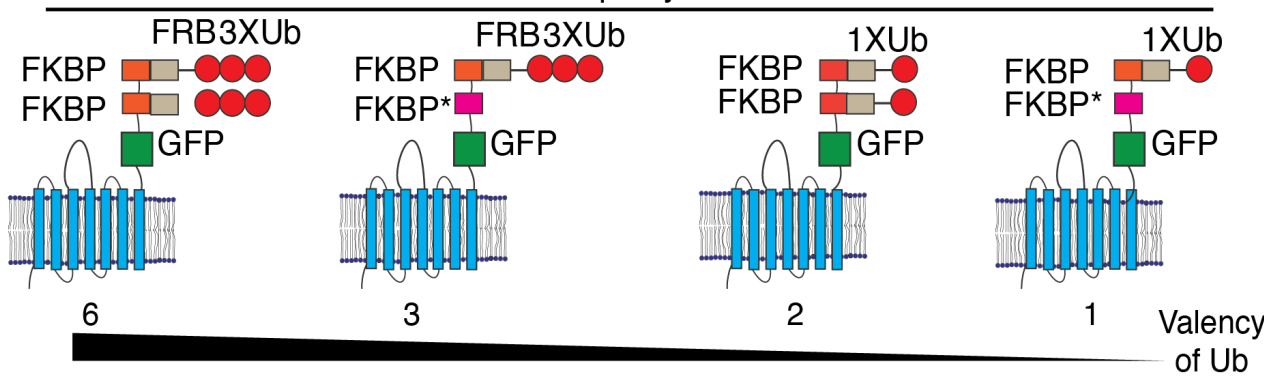

D.

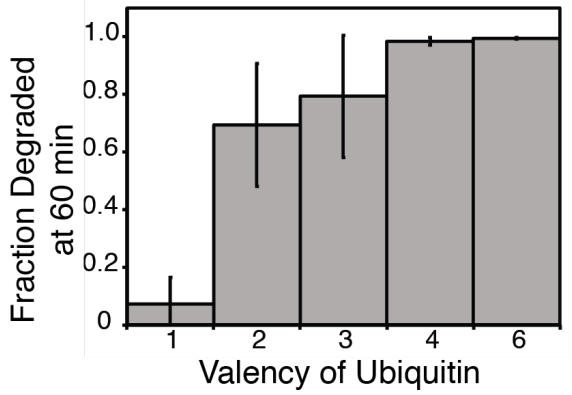

F.

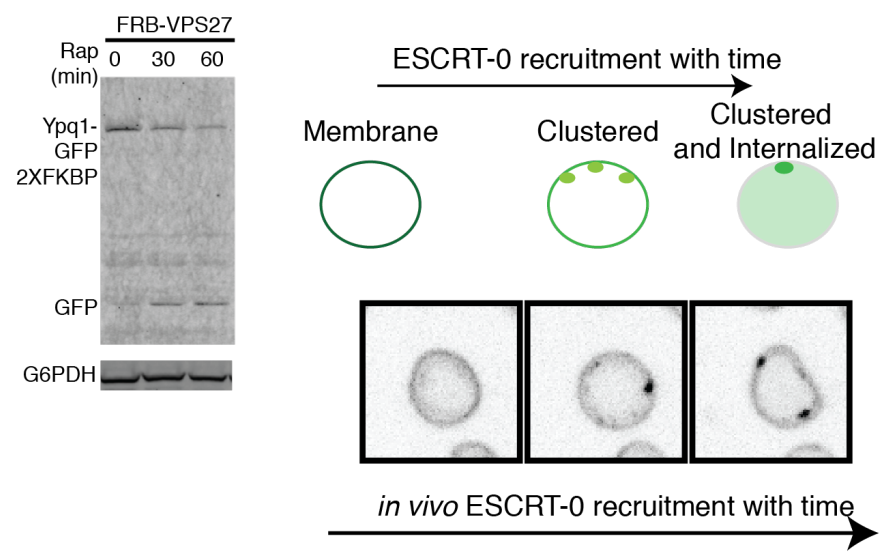

G. Competition by monovalency

Mono-Ub $(\mu \mathrm{M})$

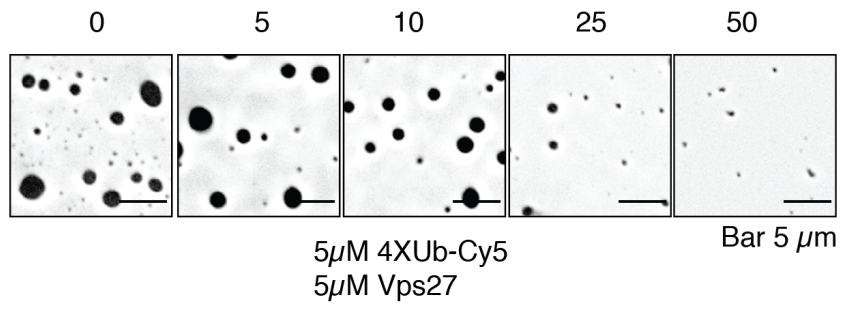

$0.5 \mu \mathrm{M}$ Vps27

$0.5 \mu \mathrm{M}$ Hse1

$0.5 \mu \mathrm{M} 4 \mathrm{XUb}-\mathrm{Cy} 5 \quad 0.5 \mu \mathrm{M} 4 \mathrm{XUb}-\mathrm{Cy} 5$

$0 \mathrm{~min}$

$40 \mathrm{~min}$

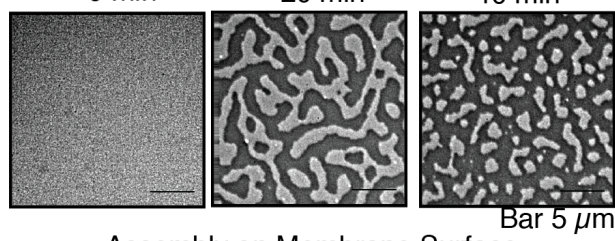

Assembly on Membrane Surface

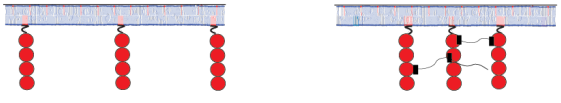

Clustered through ESCRT-0

$\begin{array}{ll}\text { Ni-NTA lipid } & \text {, ESCRT-0 } \\ =\text { His-6 } & \text { ub-binding motif } \\ \text { oubiquitin }\end{array}$

$\mathrm{H}$.

DIC

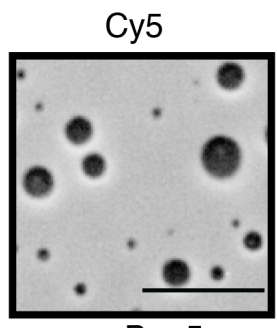

$5 \mu \mathrm{M} 4 \mathrm{XUb}-\mathrm{Cy} 5$ Bar $5 \mu \mathrm{m}$

$5 \mu \mathrm{M}$ Vps27

$5 \mu \mathrm{M}$ Hse1 
Figure 2. Multivalent interactions drive cargo clustering and sorting at the vacuole. A) Models of Ypq1 the FRB constructs used in B-D. B) Localization of Yqp1 constructs with and without rapamycin addition for 60 minutes. FKBP* contains mutations on the FRB-binding sites. C) Immunoblots for GFP after addition of Rapamycin for the indicated times. The free GFP band appears and increases in intensity as the full-length protein gets internalizes into the vacuole lumen. D) Quantification of the fraction GFP degraded into the vacuole lumen, at 60 minutes, from the data in C). E) Recruiting FRB-Vps27 onto the vacuolar cargo Ypq1-GFP-2XFKBP induces degradation of the cargo. E) Recruitment of FRB-Vps27 onto the vacuole via binding to Ypq1-GFP-2XFKBP also induces formation of foci on the surface of the vacuole. F) Assembly of Vps27-Hse1 and Cy5 labeled His64XUb on supported lipid bilayers consisting of 2\% Ni-NTA PE. Bottom model-figure shows how $4 \mathrm{X}-\mathrm{Ub}$ is associated with lipid bilayers. Model of ubiquitin binding motifs represent only a fraction of the ESCRT-0 complex. G) Competition experiment of the condensates with the inclusion of mono-ubiquitin at the mentioned concentrations. Protein components were combined together and fluorescence of $\mathrm{Cy} 5$ imaged after 2 hours at room temperature. Experiments were performed in $25 \mathrm{mM}$ Bis-Tris pH 6.5, $150 \mathrm{mM} \mathrm{NaCl}$. H) Recombinant Vps27 and Hse1 were incubated with 4X-Ub-Cy5 and imaged after 2 hours at room temperature, showing DIC image on the left and fluorescence (of Cy5) on the right. Experiments were performed in $25 \mathrm{mM}$ Bis-Tris $\mathrm{pH} 6.5,150 \mathrm{mM} \mathrm{NaCl}$. 


\section{Figure 3}

A.

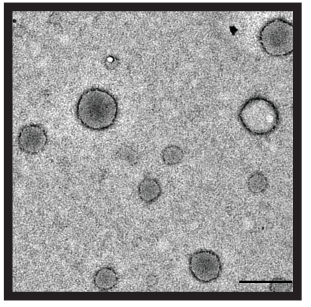

Bar $200 \mathrm{~nm}$

B.

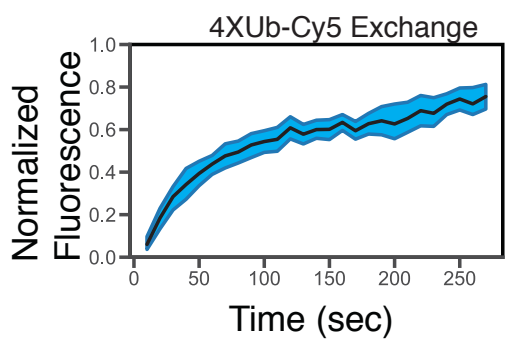

D.

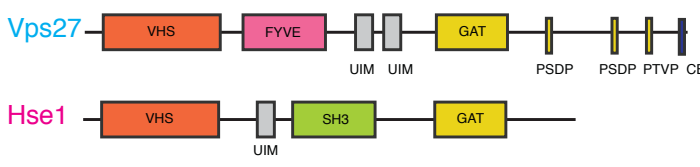

$\mathrm{F}$.
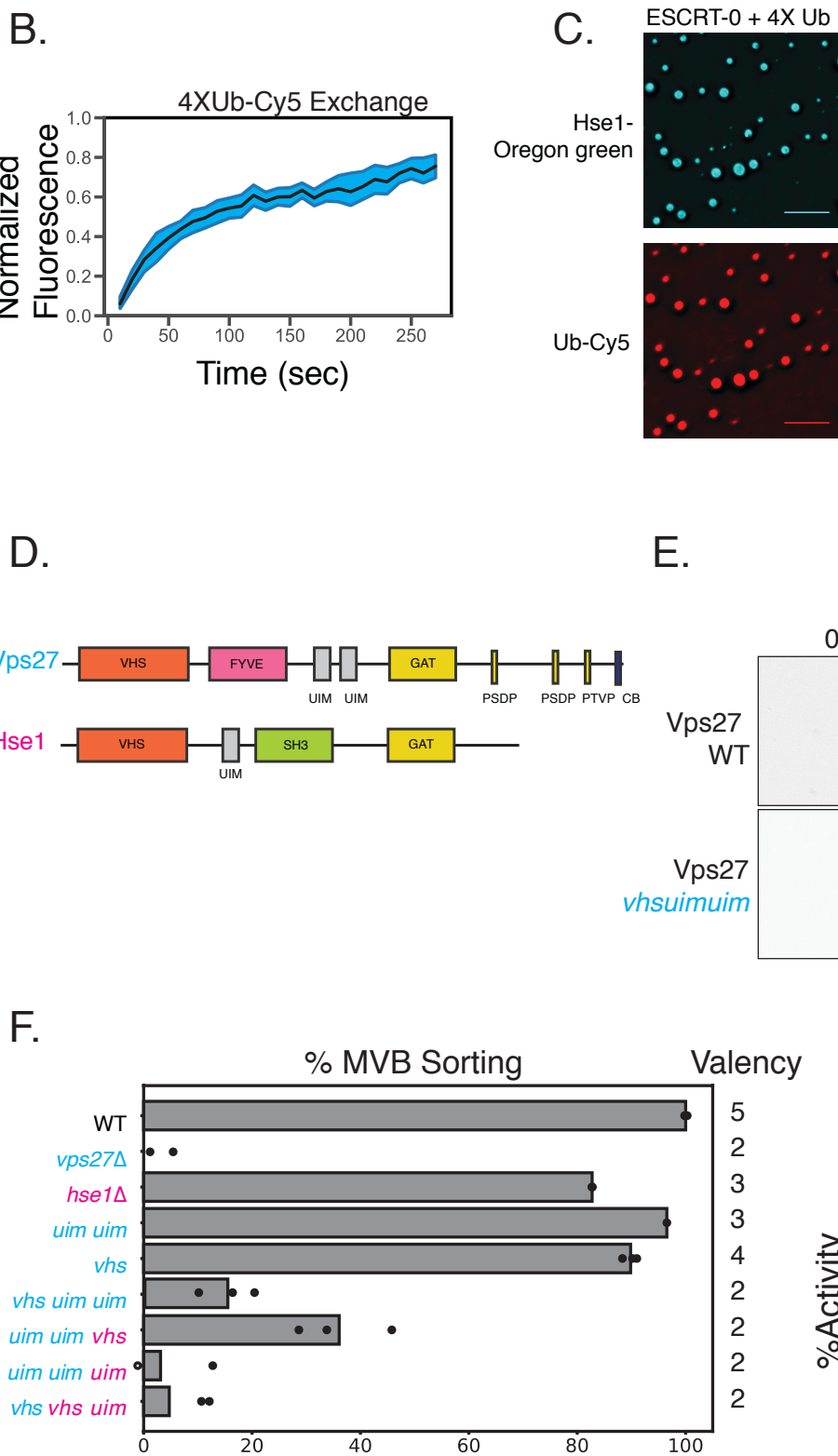

E.

F.
Electron Microscopy

250 nM His6-4XUb + 250 nM ESCRT-0

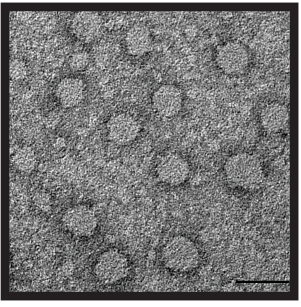

Bar 200 nm

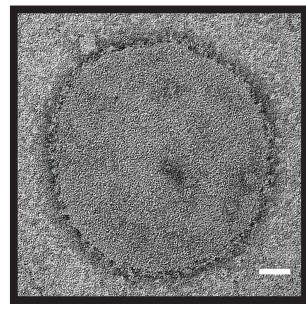

Bar $50 \mathrm{~nm}$

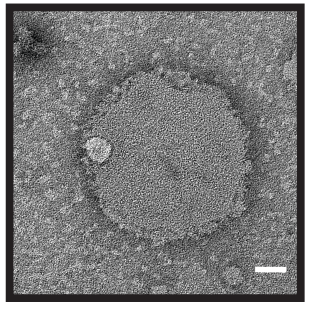

Bar $50 \mathrm{~nm}$
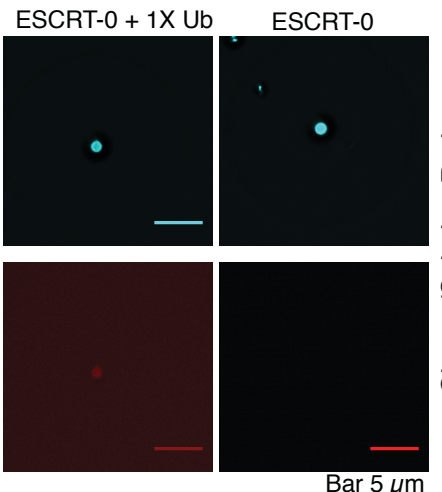

Ub Recruitment to condensates
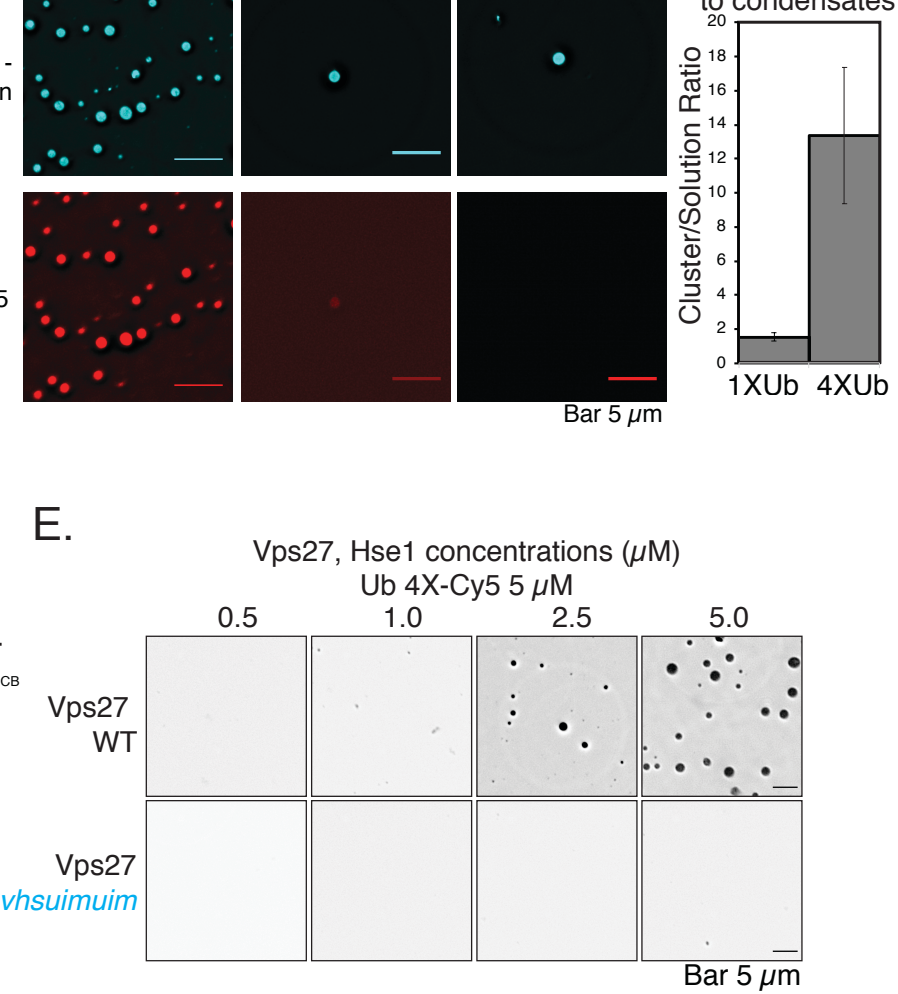

Bar $5 \mu \mathrm{m}$

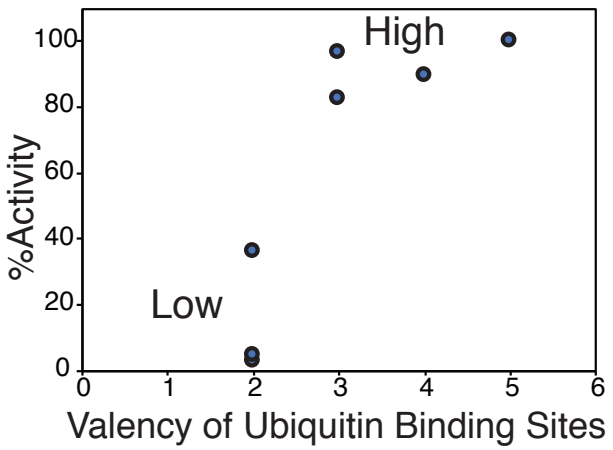


Figure 3. Multivalent interactions drive cargo sorting reactions. A) Electron microscopy images of the ESCRT-0 complex (Vps27/Hse1) with His6-4X-Ub. The structures form variable sizes distributions and are spherical in nature. Electron microscopy was performed at $25 \mathrm{mM}$ Hepes $\mathrm{pH} 7.5,150 \mathrm{mM} \mathrm{NaCl}$. B) Fluorescence recovery after photobleaching (FRAP) of 4XUb-Cy5. Three whole droplets were bleached to obtain the normalized fluorescence recovery curves. C) Purified Vps27 and Hse1 were incubated with $4 \mathrm{X}-\mathrm{Ub}$ and imaged after 2 hours at room temperature. $4 \mathrm{X}-\mathrm{Ub}$ contains a His 6 tag at the Nterminus and also a cysteine that has been labeled with a Cy5 dye for visualization. Hse 1 was labeled with Oregon-green maleimide. Right figure is a quantification of the level of mono-ubiquitin-Cy5 or tetraubiquitin-Cy5 recruitment in droplets made from respective proteins. D) Domain organization of the ESCRT-0 proteins Vps27 and Hse1. Vps27 contains three ubiquitin binding domains (a VHS and two UIMs), while Hse 1 contains two (a VHS and a UIM motif). E) Condensate formation with WT Vps27 or the Vps27 vhsuimuim mutant, in the presence of Hse 1 and $4 \mathrm{XUb}-\mathrm{Cy} 5$. Experiments were performed in $25 \mathrm{mM}$ Bis-tris pH6.5, $150 \mathrm{mM} \mathrm{NaCl}$. The mutant Vps27 contains mutations in the VHS domain and mutations in the UIM motifs that abrogate ubiquitin binding. F) Mup1pHluorin sorting assay through flow cytometry with different ESCRT-0 mutants. Mutations were made in the ESCRT-0 components Vps27 and Hse 1 at their VHS and UIM motifs. Cyan italics represent mutations in Vps27, and the magenta italics represent mutations in Hse1. Numbers on the right of the panel represent the number of ubiquitin binding sites present in the ESCRT-0 complex in the various mutants. Dots in the bars represent independent experiments, while the bars represent average sorting from three independent experiments. Right: Sorting activity (as obtained from F) for Mup1-phluorin sorting as a function of ubiquitin valency in ESCRT-0. 


\section{Figure 4}
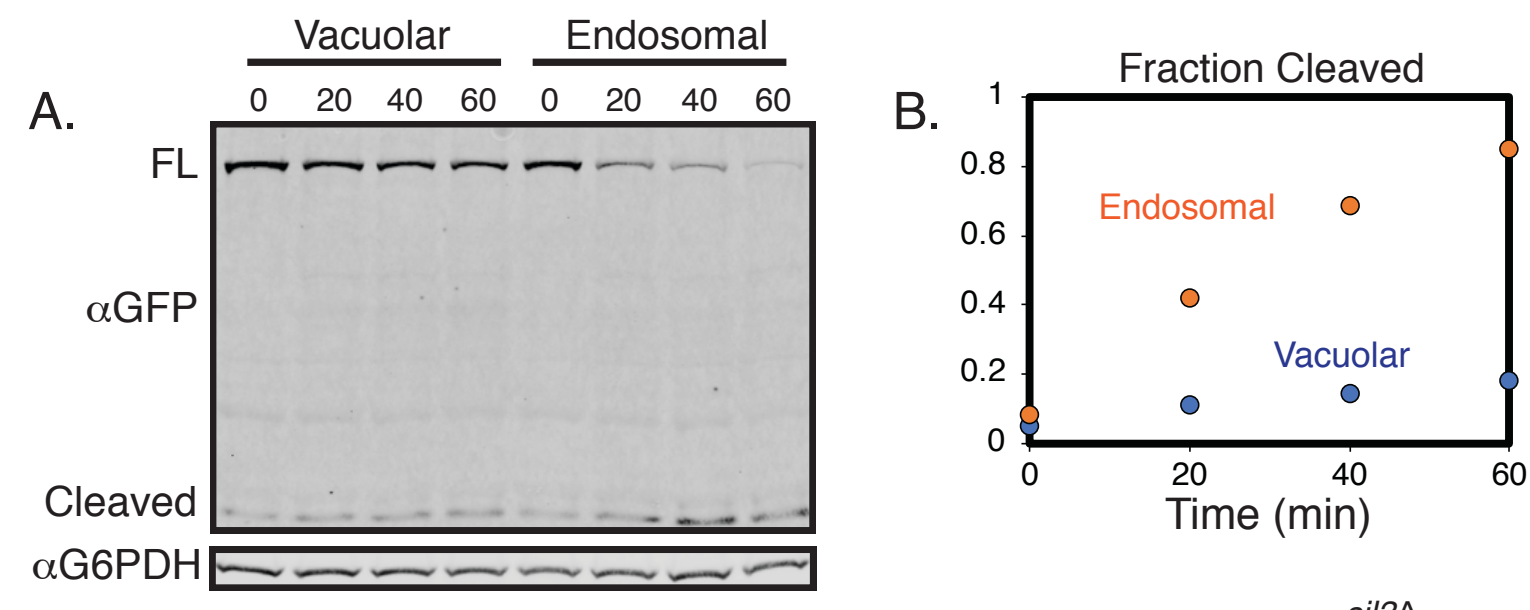

C.
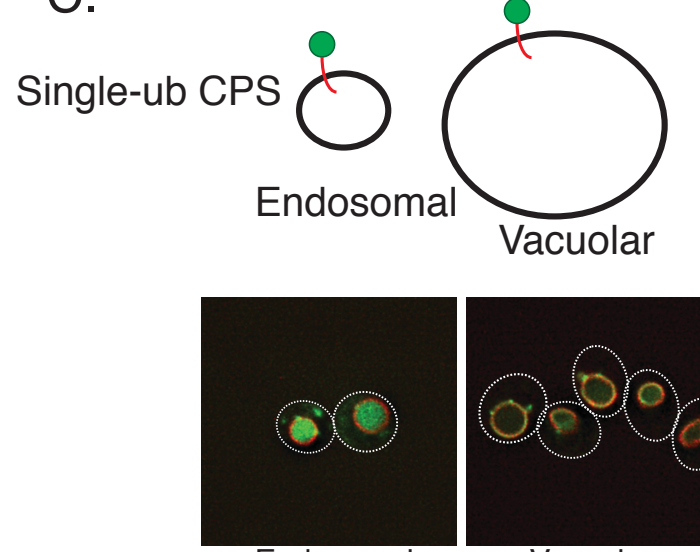

Endosomal

E.

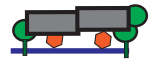

Higher density of molecules on lower surface area

Degree of poly-ubiquitination

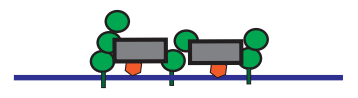

Lower density

$0, \mathrm{I}$, and II

Recruitment

and condensate formation to cluster cargo

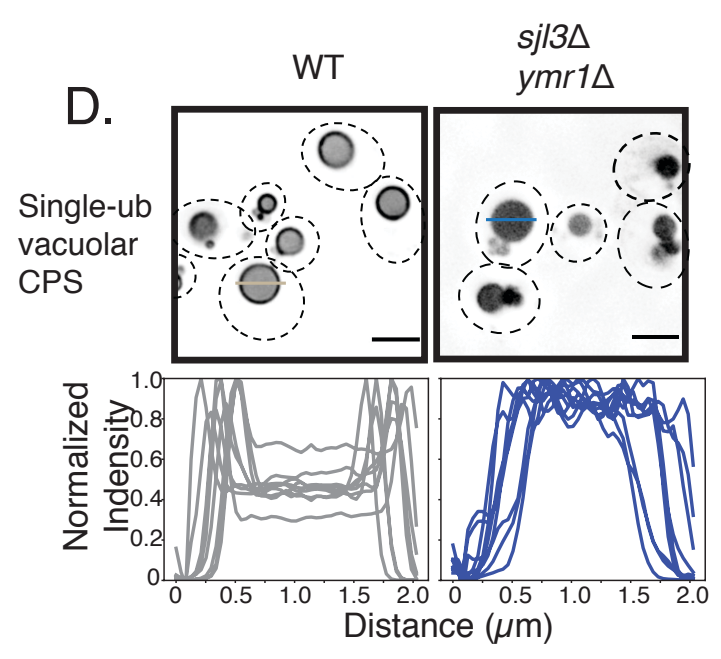

ii. ESCRT-III Assembly and Membrane Invagination

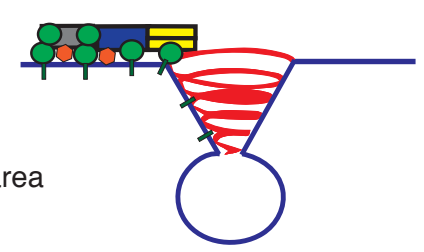

iii. ESCRT-III Disassembly and Membrane Scission
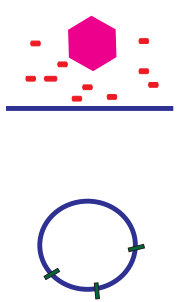

ESCRT-0

ESCRT-I ESCRT-II

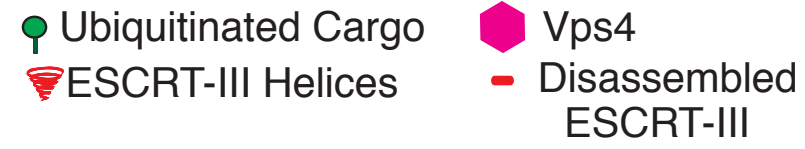


Figure 4. Differential dependence of valency on cargo sorting on endosomal and vacuolar cargo. A) Kinetics of the degradation of $\mathrm{UB}^{\mathrm{KR}}-\mathrm{GFP}-\mathrm{CPS}^{\mathrm{KR}}$ (endosomal) and $\mathrm{UB}^{\mathrm{KR}}$-GFP-alp-CPS ${ }^{\mathrm{KR}}$ (vacuolar) proteins upon induction of expression for 20 minutes and further inhibition of translation with cycloheximide addition. The 0 timepoint represents 20 minutes of GAL induction with no cyloheximide. B) Quantification of the fraction of GFP cleaved of the data from A). C) Localization of the UB ${ }^{\mathrm{KR}}-$ GFP-CPS ${ }^{\mathrm{KR}}$ (endosomal) and the $\mathrm{UB}^{\mathrm{KR}}$-GFP-alp-CPS ${ }^{\mathrm{KR}}$ (vacuolar) constructs after inducing the expression for 20 minutes through the GAL promoter and then stopping translation through cycloheximide addition for 30 minutes. D) Localization of the indicated $\mathrm{UB}^{\mathrm{KR}}$-GFP-alp-CPS ${ }^{\mathrm{KR}}$ (vacuolar cargo) in the phosphatase mutant strains in the phosphatidyl-inositol-3P phosphatase mutant $y m r l \Delta s j l 3 \Delta$. Intensity profiles on the bottom represent line scan across the vacuoles. Representative lines are shown across two vacuoles (gray and blue lines) in the microscopy images. Scale bars are $2 \mu \mathrm{m}$ long. E) Models suggesting how variation of ESCRT and cargo density affects recruitment and condensation of early ESCRT and substrate on the surface of membranes. The condensation of early players in the pathway may nucleate downstream assembly, leading to ESCRT-III polymerization and membrane budding.

Movie 1: In vivo visualization of Ypq1-GFP-2X-FKBP upon addition of rapamycin. Yeast cells were treated with $1 \mu \mathrm{g} / \mathrm{mL}$ of Rapamycin and imaged over the timeframe indicated in the movie. 


\section{Supplementary Figures:}

\section{Figure S1}

A.
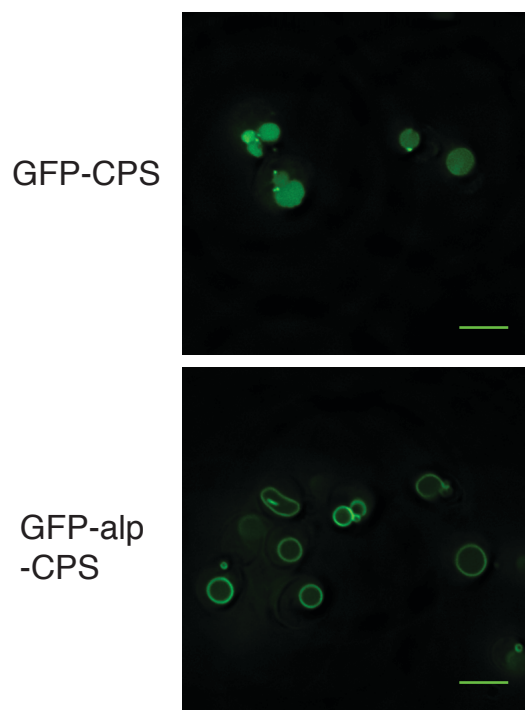

B.

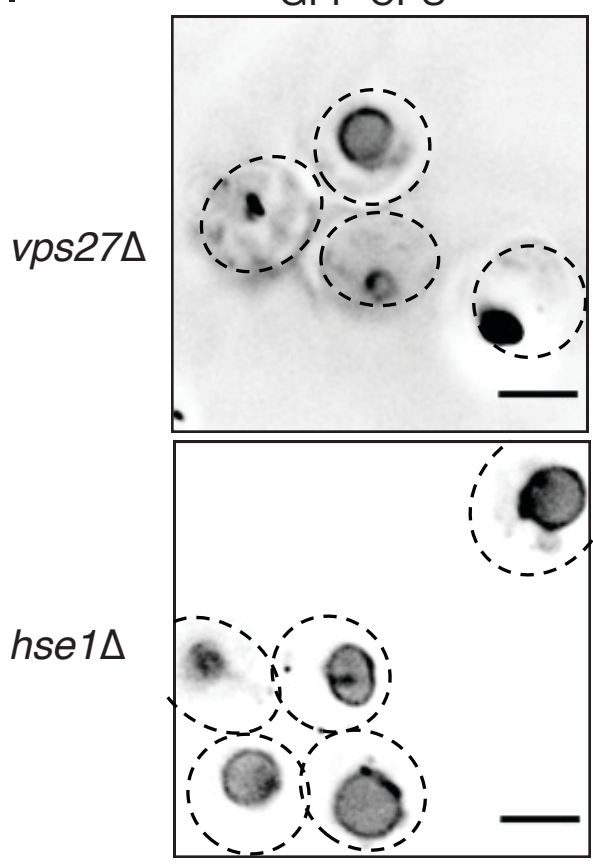

Vph1-mcherry
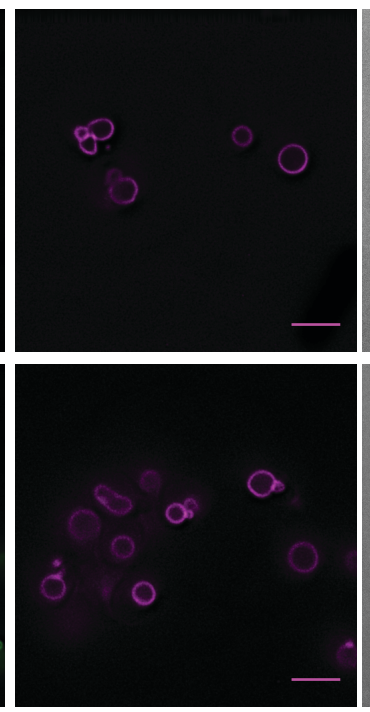

DIC
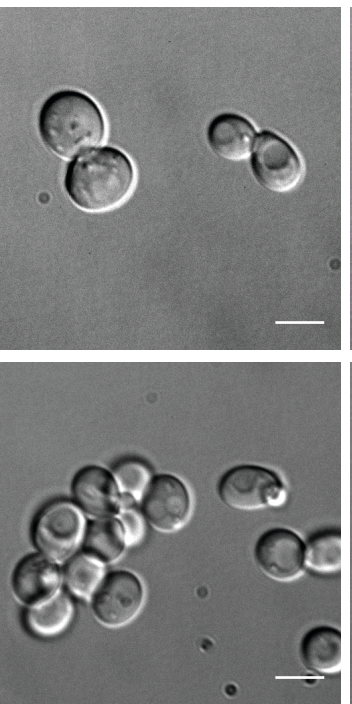

Merge

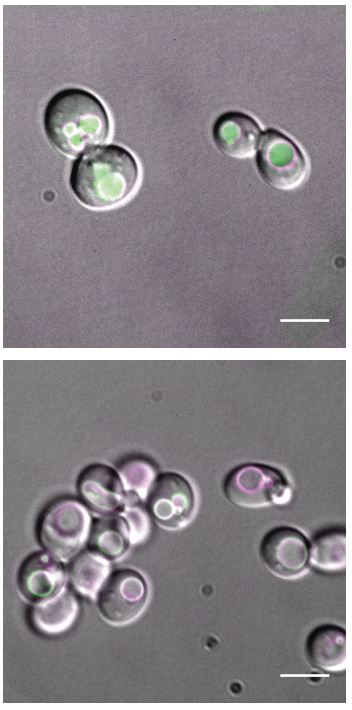

S1. Localization of endosomal and vacuolar GFP-CPS molecules. A) GFP-CPS or GFP-alpCPS in wild-type strains expressing Vph1-mcherry. Vph1 is a vacuolar membrane protein. Scale 
bars are $5 \mu \mathrm{m}$. B) Localization of different GFP-CPS constructs in the ESCRT-0 components vps $27 \Delta$ and $h$ se $1 \Delta$ strains. Scale bars are $2 \mu \mathrm{m}$ each.

Figure S2

Ub-GFP-ALP-CPS (vacuolar)
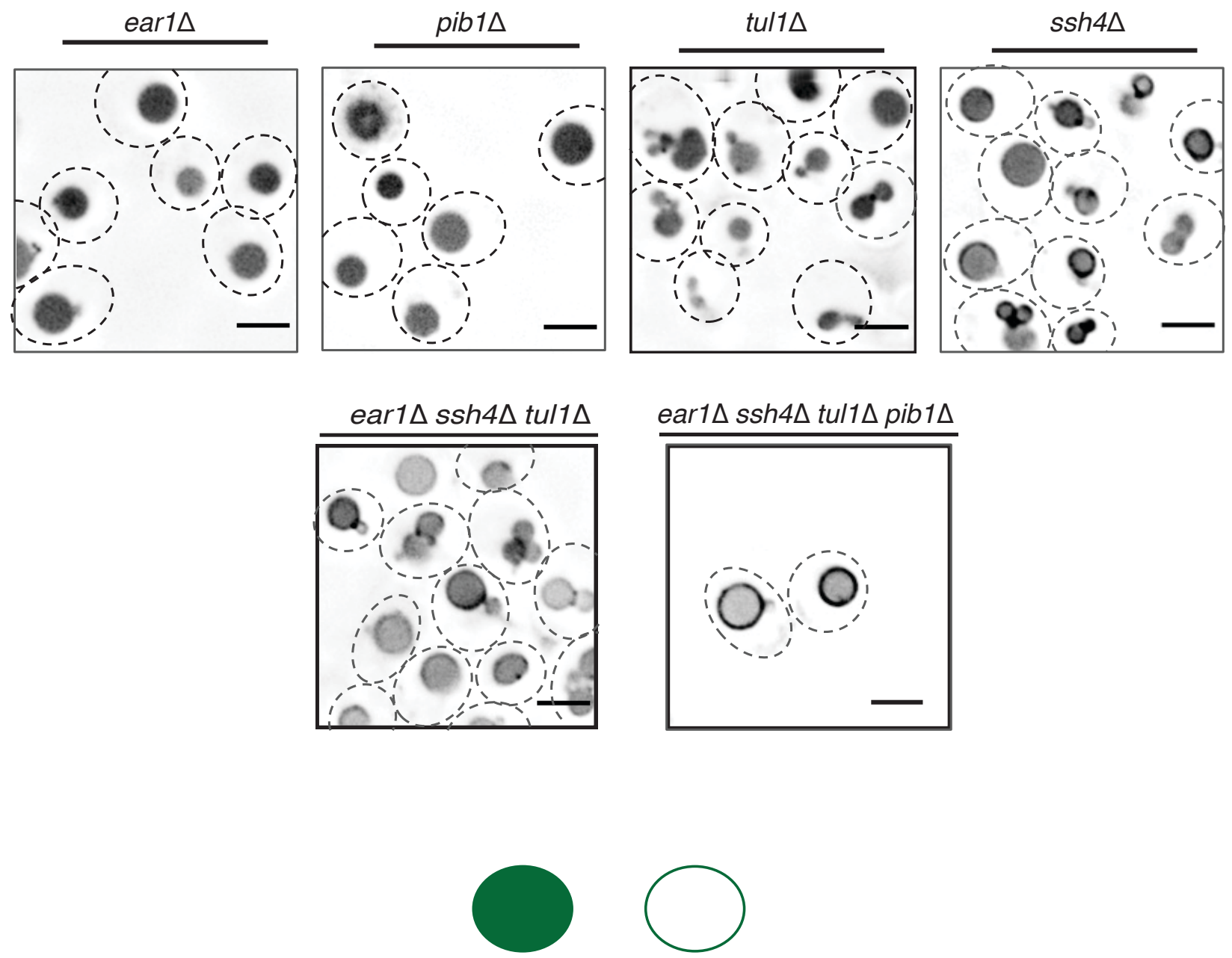

vacuole lumen

vacuole membrane

S2. Poly-ubiquitination is critical for vacuolar sorting of ESCRT cargo. Localization of Ub-

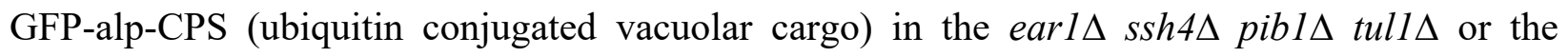
individual strains. Scale bars are $2 \mu \mathrm{m}$ each. 


\section{Figure S3}

A.

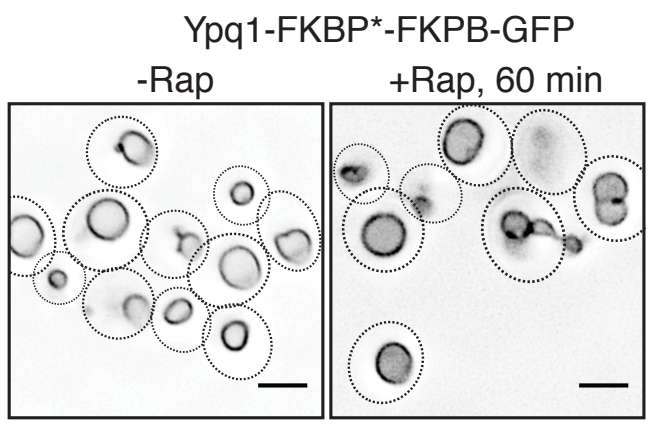

FRB-3XUb

(P. 60 min

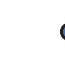

$\stackrel{\circ}{\stackrel{0}{\text { FRB- }} \text { - }}$

$\underline{\text { FRB- }}$

B.

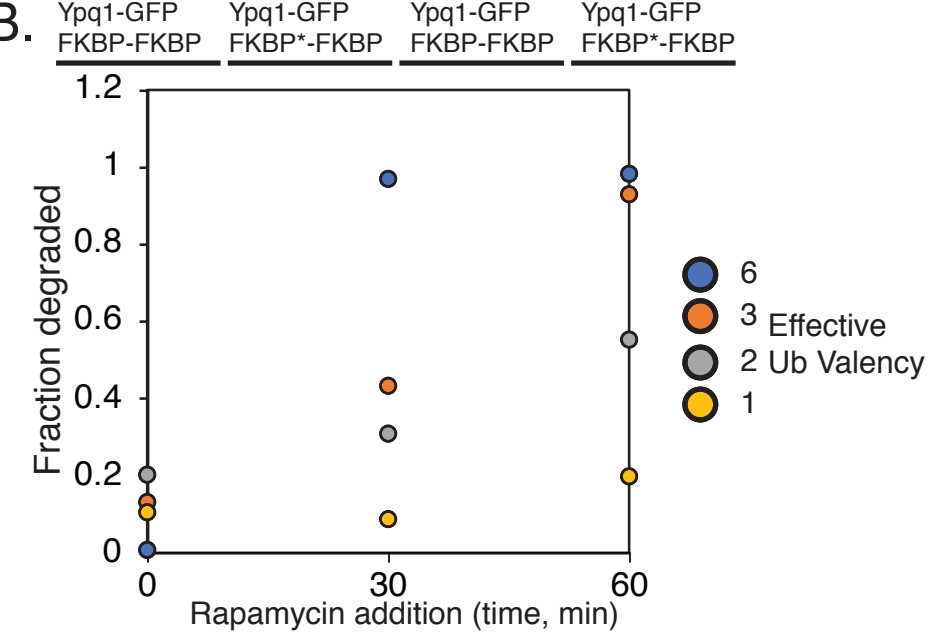

O

FRB-

1X-Ub

FBP*-FKBP

C.

Vph1-FKBP-FKBP-GFP, FRB-3XUb
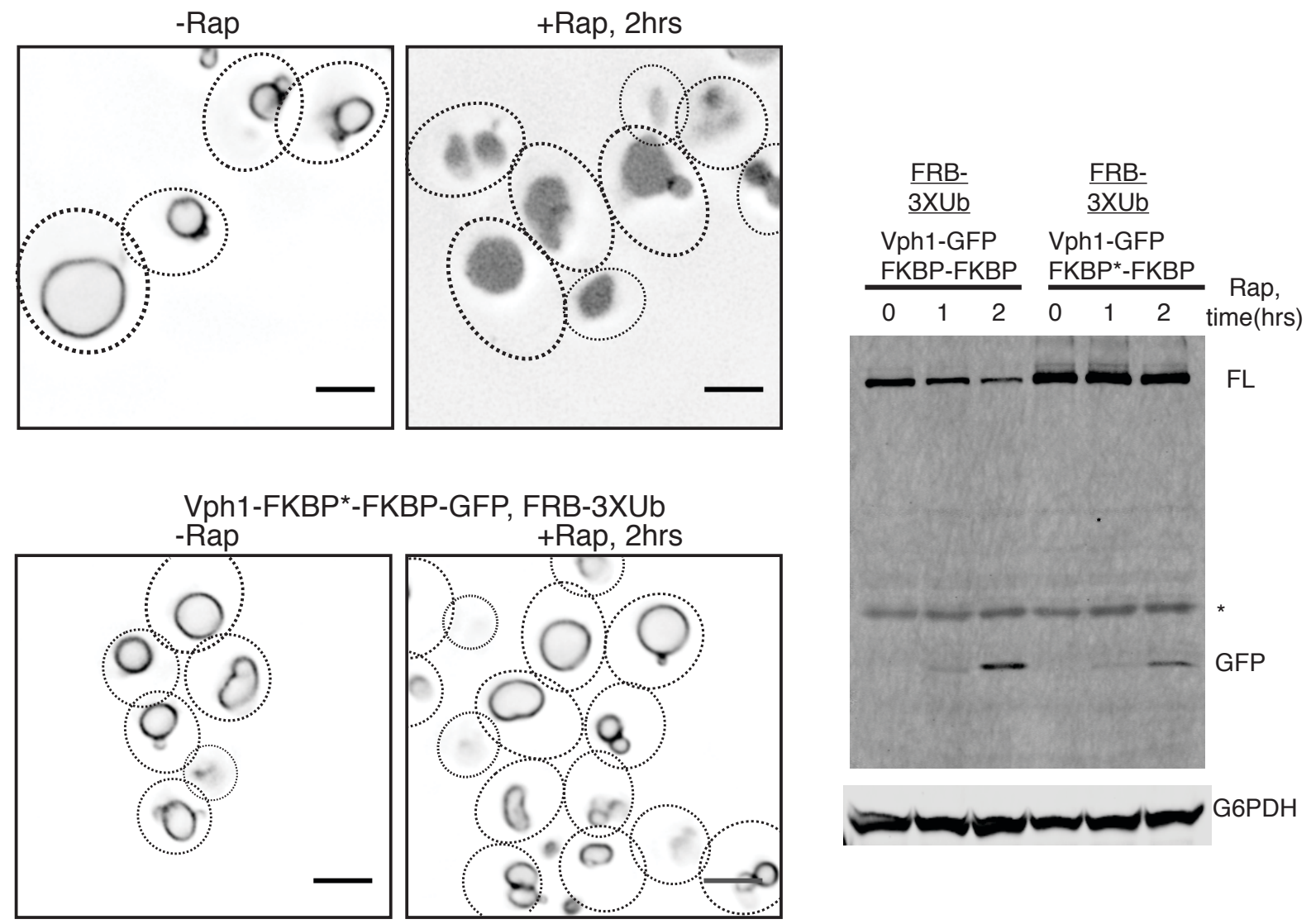

S3. Multivalent interactions of polyubiquitin and ESCRT-0 promotes cargo sorting. A) Effect of rapamycin-induced sorting of Ypq1 construct with one FKBP site mutated and can no longer interact with FRB. Strain consists of FRB-3X ubiquitin and the imaging was done before adding 
rapamycin and after 60 minutes of adding rapamycin. B) Kinetics of different Ypq1-GFP-FKBP molecules in the presence of different FRB-Ub molecules. Colors of circles indicate the effective valency of ubiquitin achieved from the combination of FKBP and FRB constructs. C) Vph1-GFPFKBP-FKBP constructs sorting in the presence of FRB-3XUb. Mutation in one of the FRB binding sites in FKBP inhibits sorting of the Vph1 construct, signifying the effect of multivalent interactions in sorting efficiency. Left figures are imaging experiments following GFP-tagged Vph1, and the right image is an immunoblot following GFP cleavage after adding rapamycin for the indicated times. 
Figure S4

A.

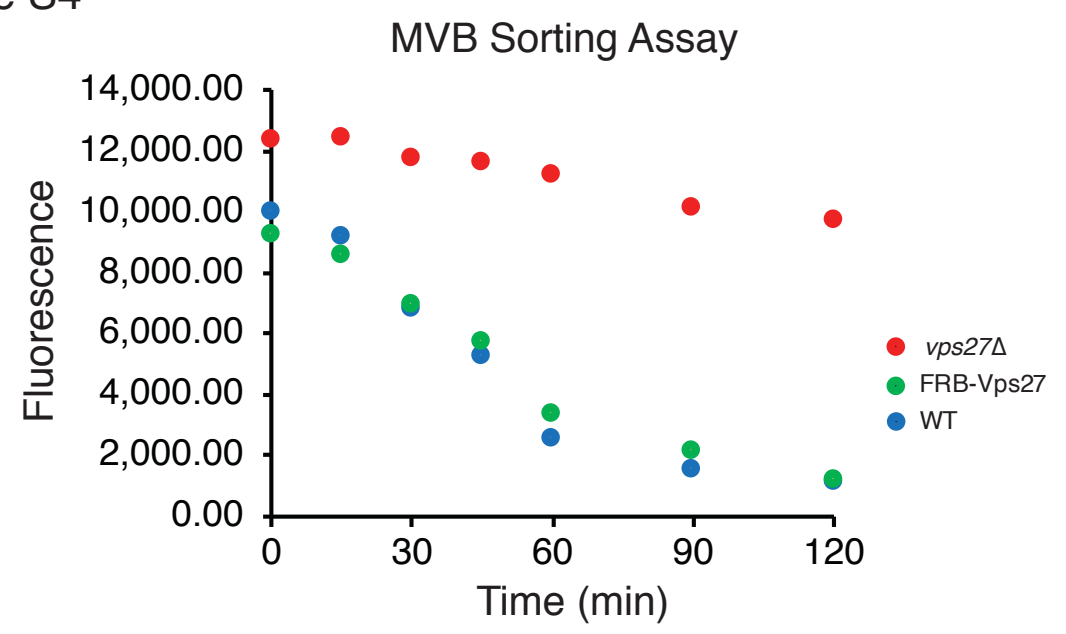

B.

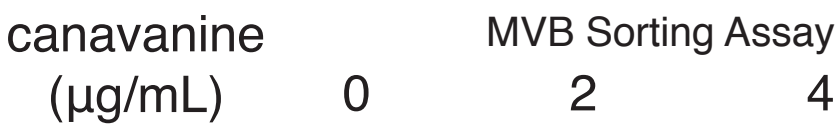

46

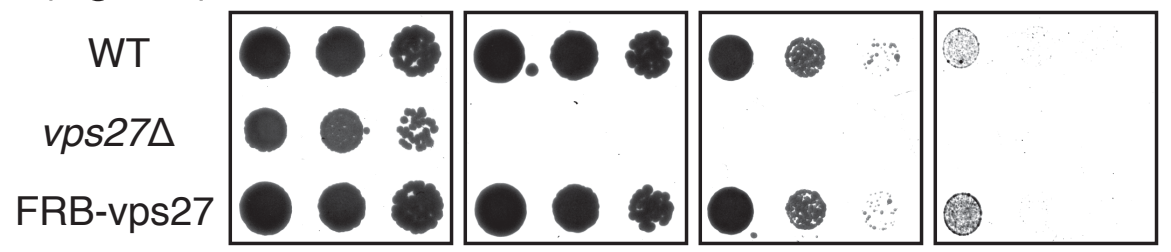

C.

+ Rap

$1 \mathrm{hr}$

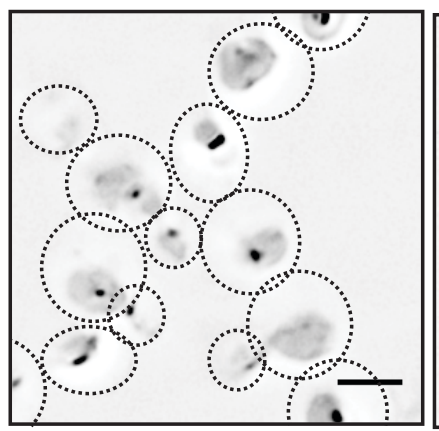

+ Rap

2 hrs
+ Rap

$2 \mathrm{hrs}$, cycloheximide at $1 \mathrm{hr}$
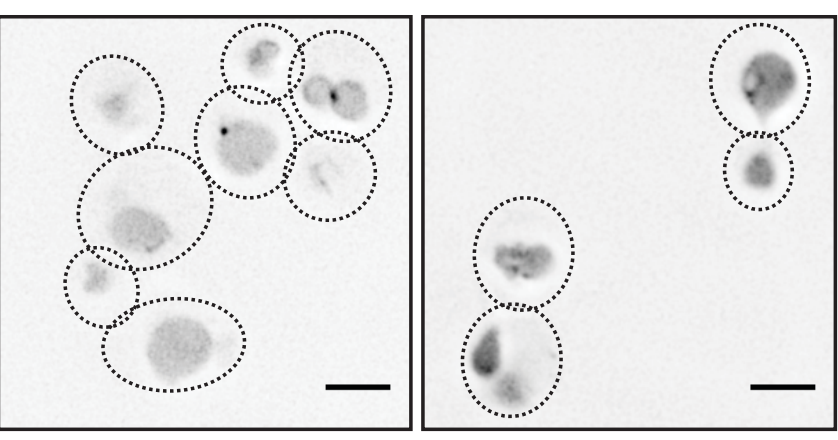

S4. Recruitment of ESCRT-0 to vacuolar membrane induces cargo clustering. A-B) FRBVps27 construct used is identical to WT Vps27 in cargo sorting assays for the cargoes Mup1 in Mup1-pHluorin sorting (A) and Can1 sorting canavanine assays (B). C) With time or with translation inhibition, the clusters induced by FRB-Vps27 recruitment disappear, presumably by internalization into the vacuolar lumen. 


\section{Figure S5}

A.

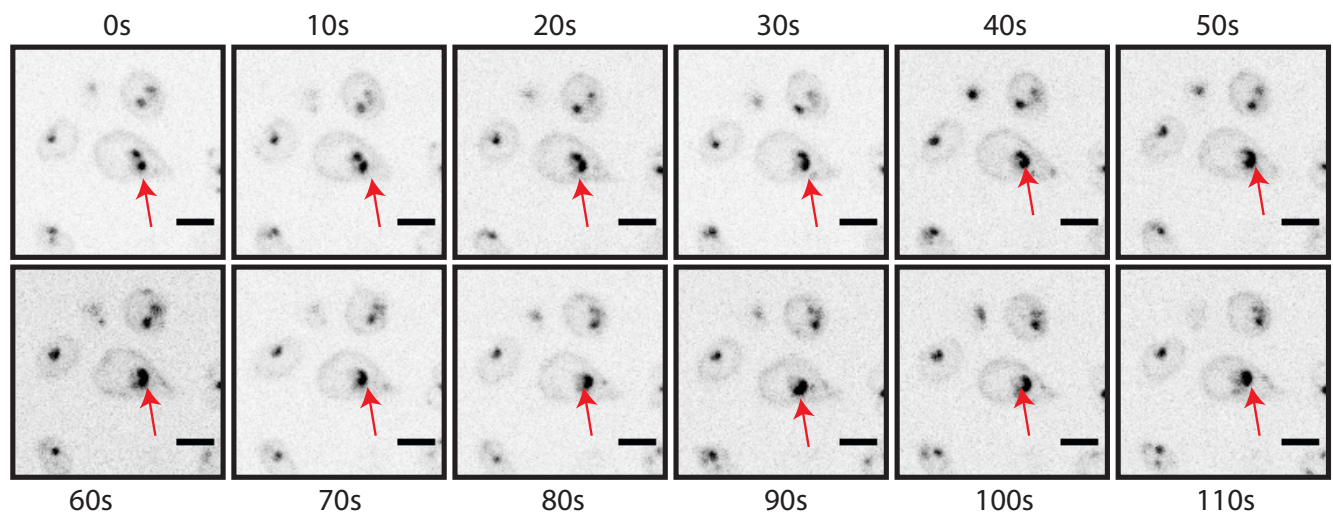

B.

Os

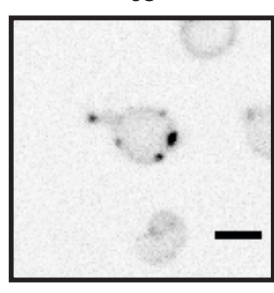

$10 \mathrm{~s}$

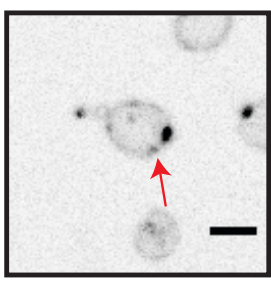

$20 \mathrm{~s}$

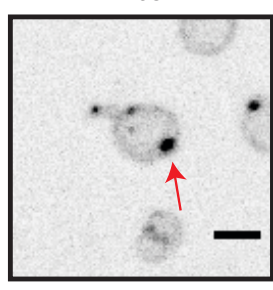

$30 \mathrm{~s}$

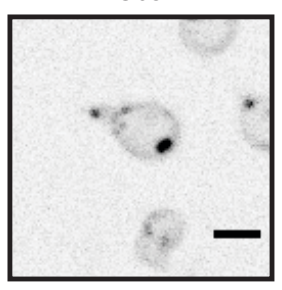

40s

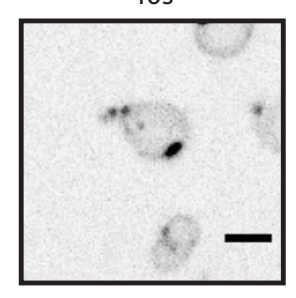

$20 \mathrm{~s}$
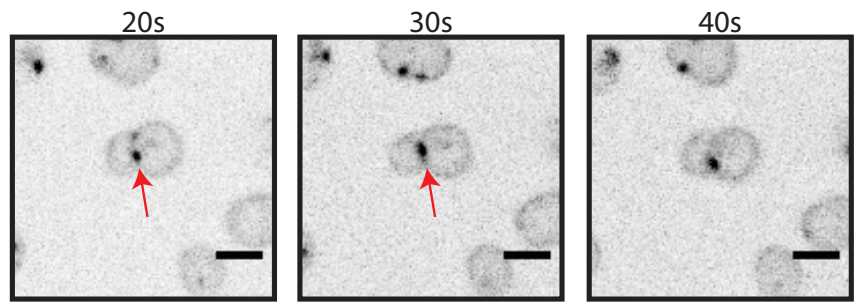

S5. In vivo dynamics of clusters of Ypq1-GFP-2XFKBP molecule induced by recruitment of

FRB-Vps27 (A-C). In these experiments, rapamycin was added for 30 minutes the imaging was performed with $10 \mathrm{~ms}$ interval. Scale bars are $2 \mu \mathrm{m}$ each. 


\section{Figure S6}

A.
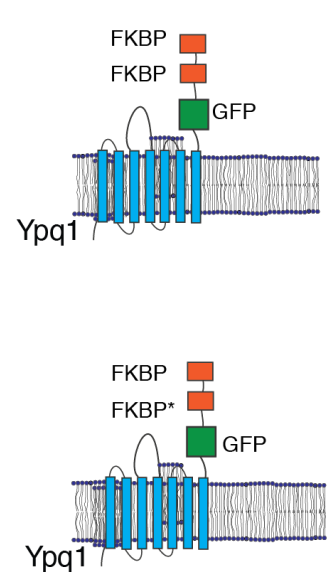

B.
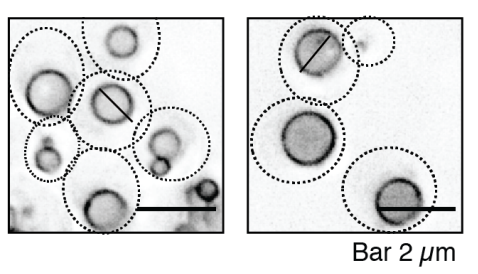

FRB-Vps27
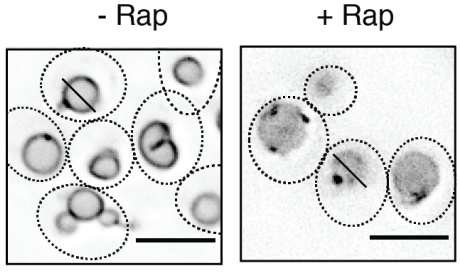
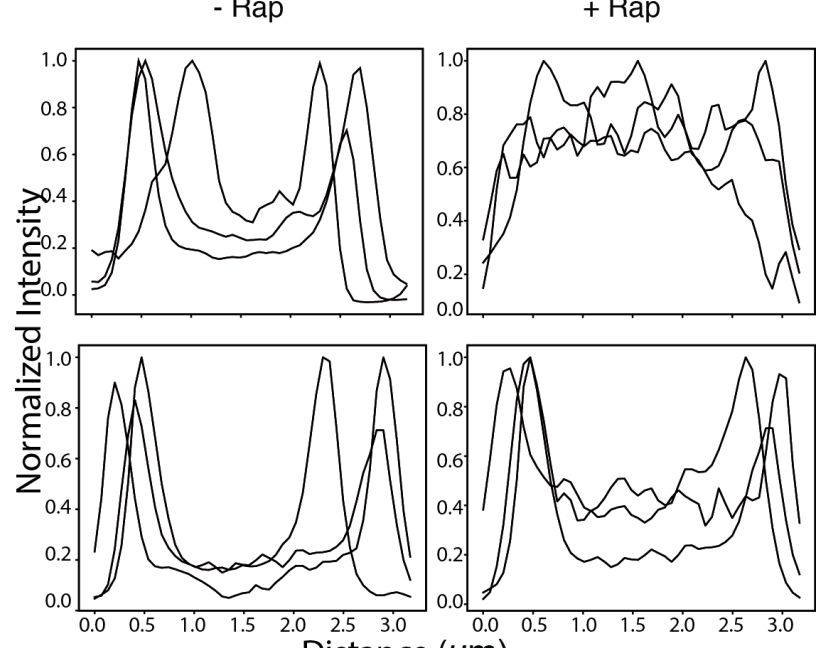

Distance $(\mu \mathrm{m})$

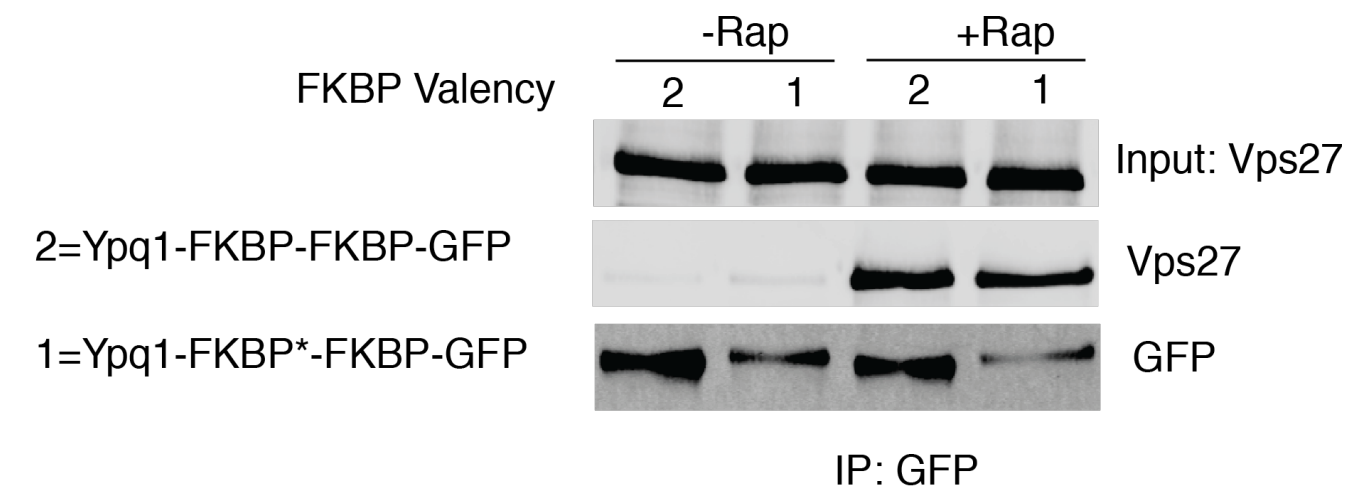

C.

Intrinsic disorder profile

Vps27

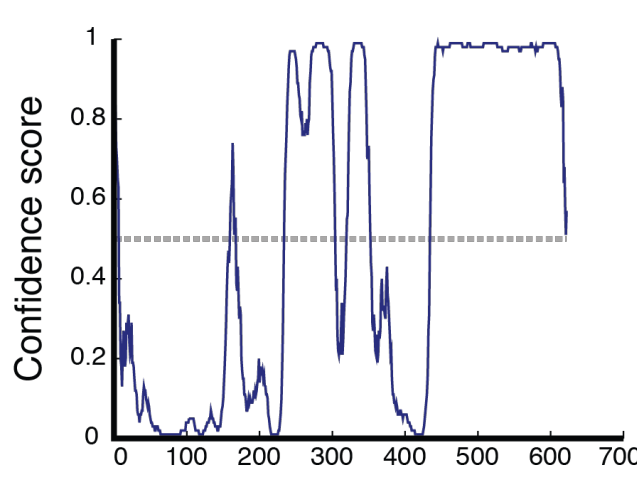

Amino acid position
Hse1

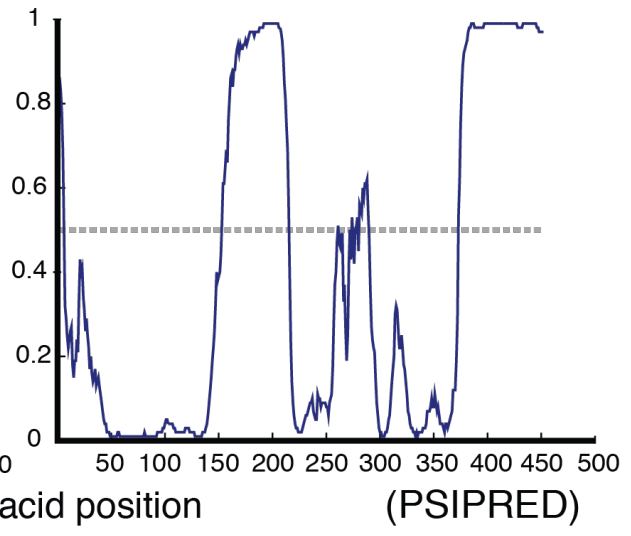

S6. Valency effect on ESCRT-0 recruitment and cargo sorting. A) Ypq1-GFP-FKBP-FKBP or one of the FKBPs mutated to abrogate FRB binding were imaged after adding rapamycin to strains 
consisting of FRB-Vps27. Cargo clustering and internalization are reduced in the strain expressing the lower valency molecule. Graphs on the right are representative normalized intensity profiles of line-scans across vacuoles from microscopy images. B) Immunoprecipitation of GFP in Ypq1GFP-FKBP-FKBP and FRB-Vps27 expressing strains and blotting for Vps27. FRB-Vps27 is still bound to Yqp1-GFP-FKBP*-FKBP in a rapamycin dependent fashion. C) Intrinsic-disorder profile obtained from DISOPRED for the ESCRT-0 components Vps27 and Hse1. 


\section{Figure S7}

A.

Solvent Effect

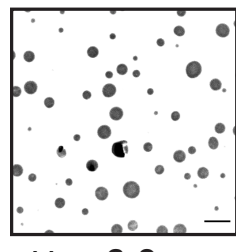

$\mathrm{pH}$

6.0

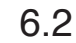

6.2

6.4

6.6

6.8

7.0

$5 \mu \mathrm{m}$

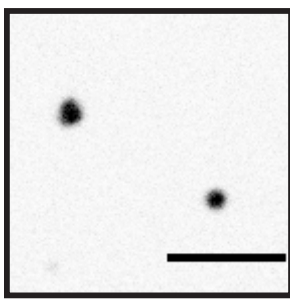

$\mathrm{pH} 7.0 \quad$ Bar $5 \mu \mathrm{m}$

B.

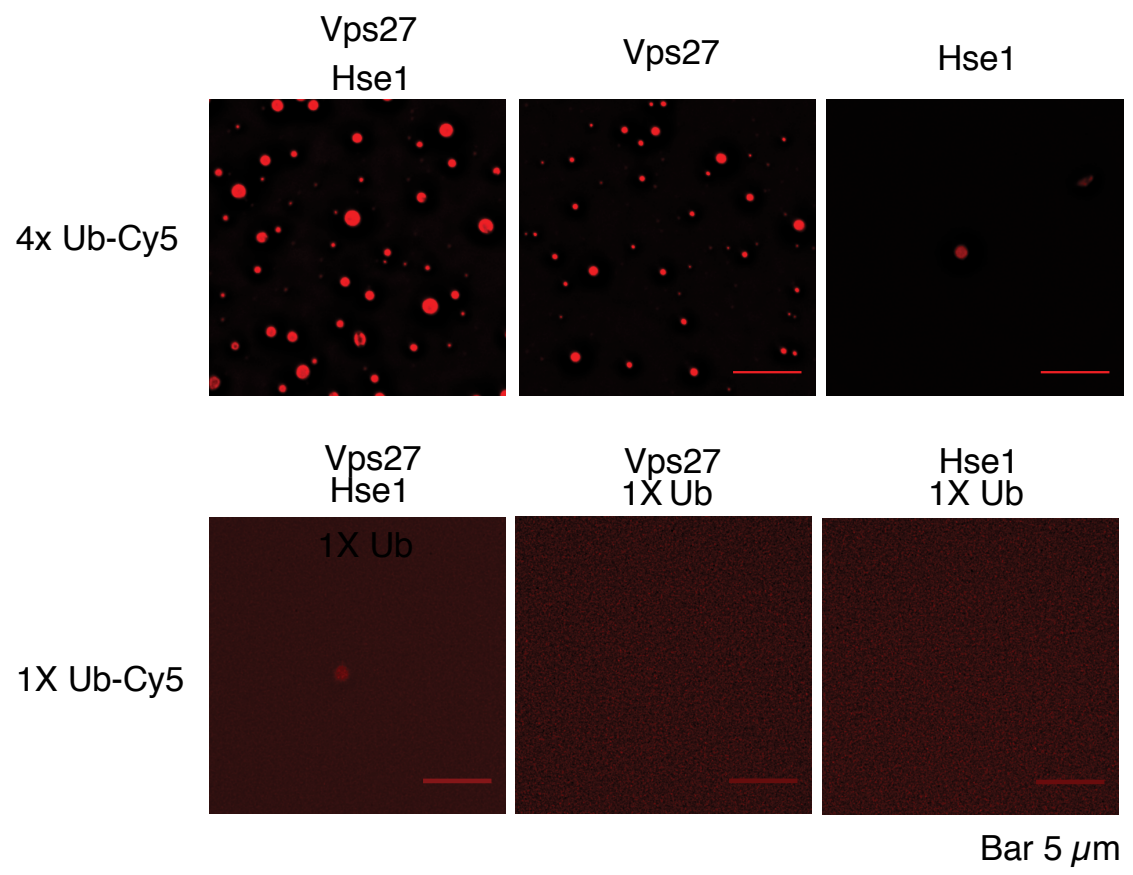

S7. Condensation of ESCRT-0 and polyubiquitin assemblies. A) Solvent effect on the condensation of ESCRT-0 and $4 \mathrm{X}$ ubiquitin. $\mathrm{pH}$ was varied by using different buffers of Bis-Tris at the indicated $\mathrm{pH}$ values. Concentration of buffer was kept at $100 \mathrm{mM}$ and $\mathrm{NaCl}$ was kept constant at $150 \mathrm{mM}$. Images were taken after two hours of assembling the components at room temperature. Bottom figure shows a zoomed-in image of the droplets at $\mathrm{pH} 7$, showing presence 
of droplets but at reduced numbers. Scale bars are $5 \mu \mathrm{m}$ each. B) Assemblies of ESCRT-0 and either $4 \mathrm{XUb}$ or $1 \mathrm{X} \mathrm{Ub}$ at the indicated compositions (either both ESCRT-0 components or singles, as represented on the top of the panels). Concentrations of the molecules were at $5 \mu \mathrm{M}$, and the buffer was $25 \mathrm{mM}$ Bis Tris $\mathrm{pH} 6.5,150 \mathrm{mM} \mathrm{NaCl}$. 


\section{Figure S8}

A.
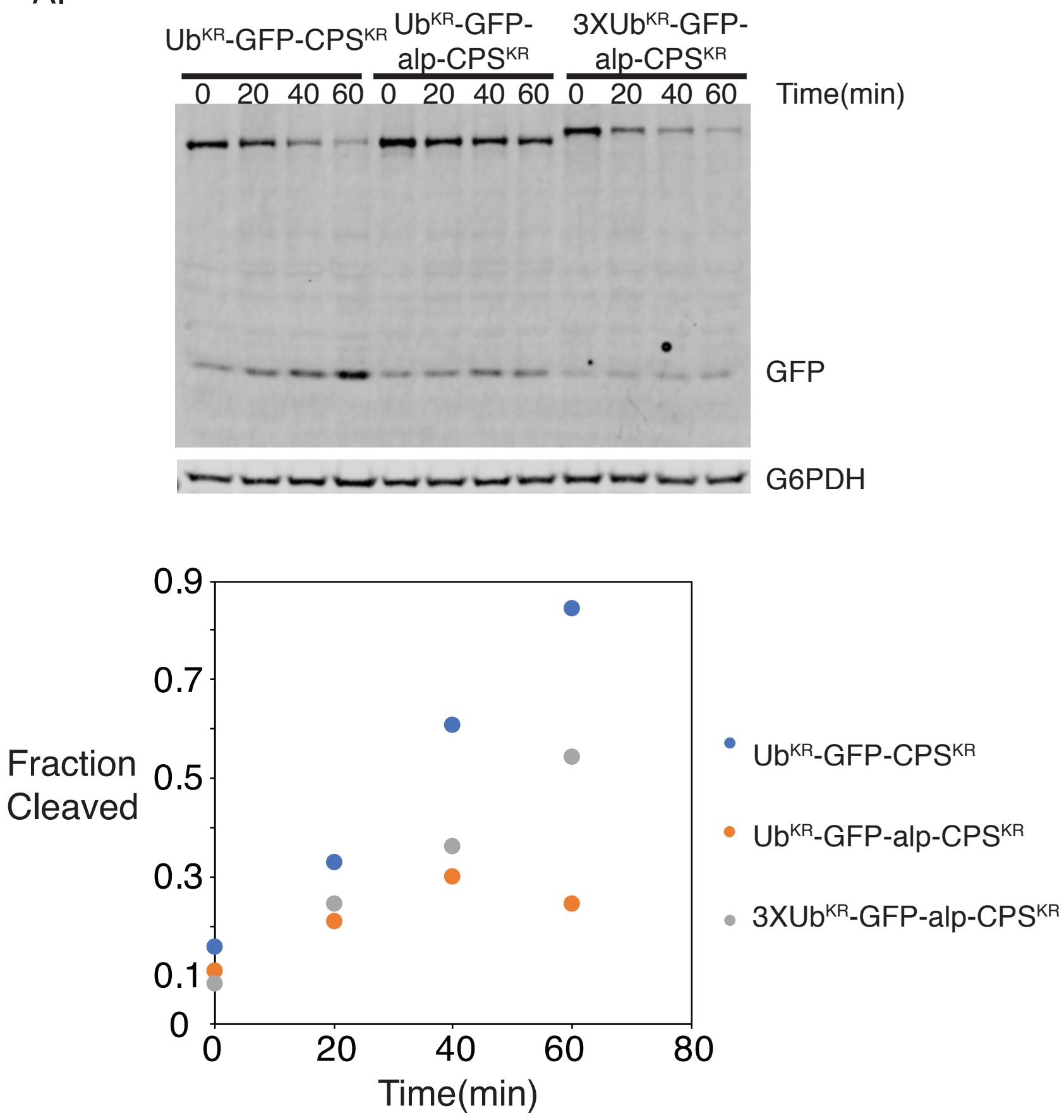

S8. Enhancing ubiquitin chain length induces vacuolar cargo sorting. A) Kinetics of the degradation of $\mathrm{Ub}^{\mathrm{KR}}$-GFP-CPS (endosomal), $\mathrm{Ub}^{\mathrm{KR}}$-GFP-alp-CPS (vacuolar), or $3 \mathrm{XUb}^{\mathrm{KR}}$-GFPalp-CPS proteins upon induction of expression for 20 minutes and further inhibition of translation 
with cycloheximide addition. The 0 timepoint represents 20 minutes of GAL induction with no cyloheximide. B) Graphical representation of the fraction degraded, with data from $\mathbf{A})$.

Figure S9

A.

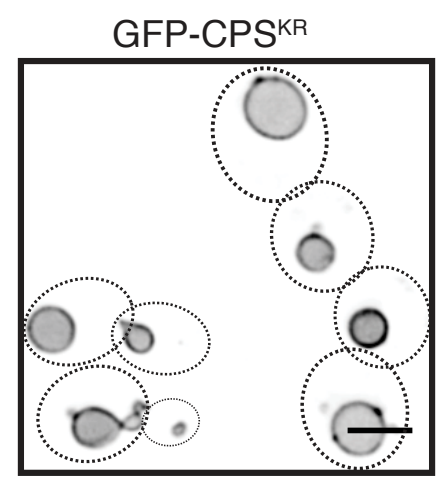

Ub-GFP-CPS ${ }^{K R}$

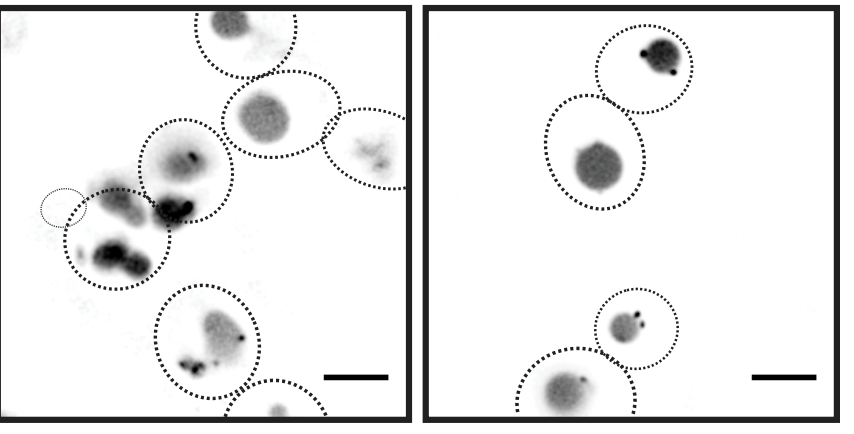

Endosomal (no alp motif)

B.

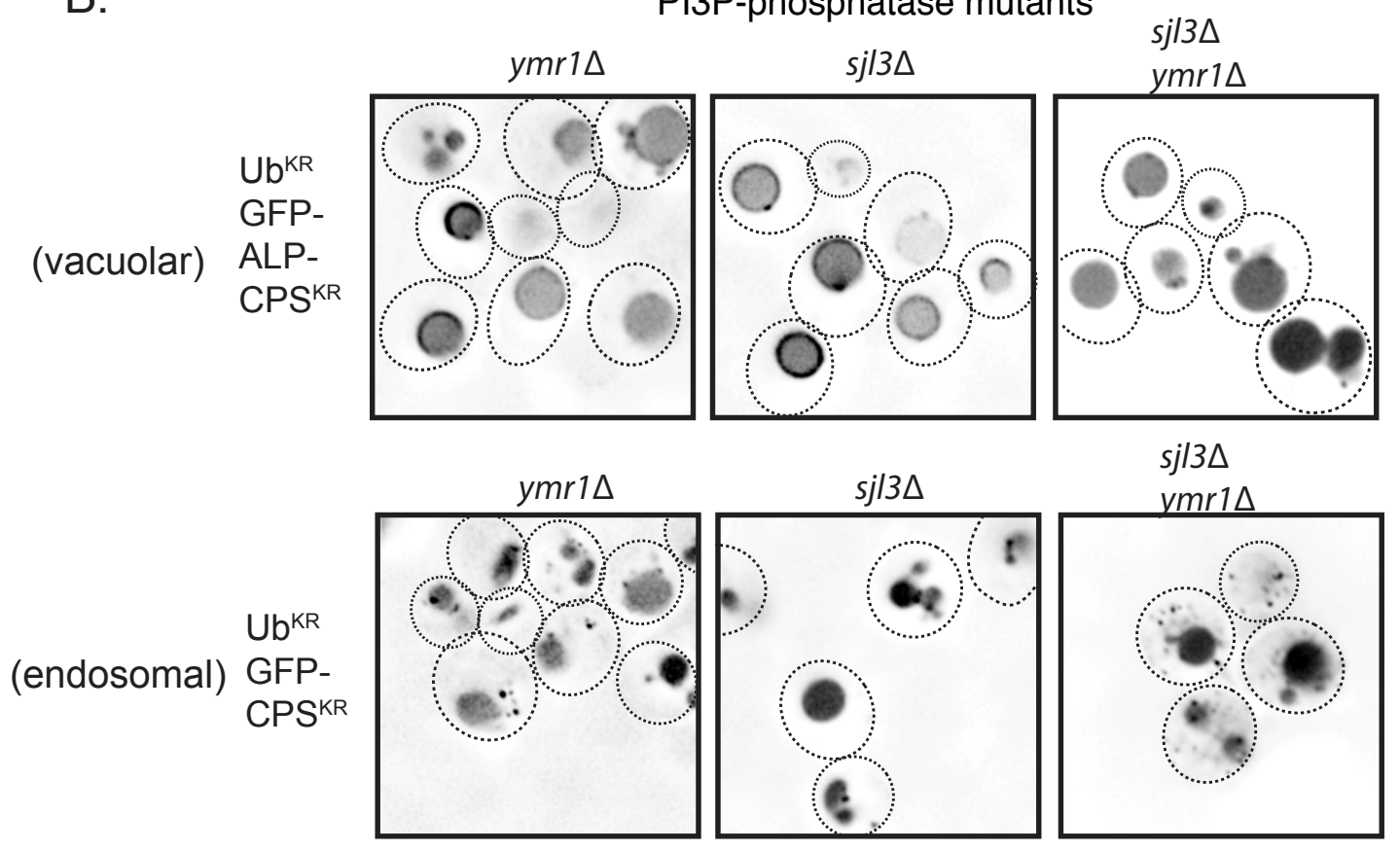

S9. A) Localization of the GFP-CPS constructs with the K8R K12R (KR) mutations, when conjugated with ubiquitin or $\mathrm{Ub}^{\mathrm{KR}}$. $\mathrm{Ub}^{\mathrm{KR}}$ has all the lysines mutated to Arg. $\mathrm{CPS}^{\mathrm{KR}}$ represents two 
bioRxiv preprint doi: https://doi.org/10.1101/2021.08.23.457352; this version posted August 23, 2021. The copyright holder for this preprint (which was not certified by peer review) is the author/funder, who has granted bioRxiv a license to display the preprint in perpetuity. It is made available under aCC-BY-NC-ND 4.0 International license.

mutations: K8R and K12R in the cytosolic region of CPS. B) Localization of the different CPS constructs in the phosphatase mutants $y m r l \Delta$ and $s j l 3 \Delta$. 


\section{Figure S10}

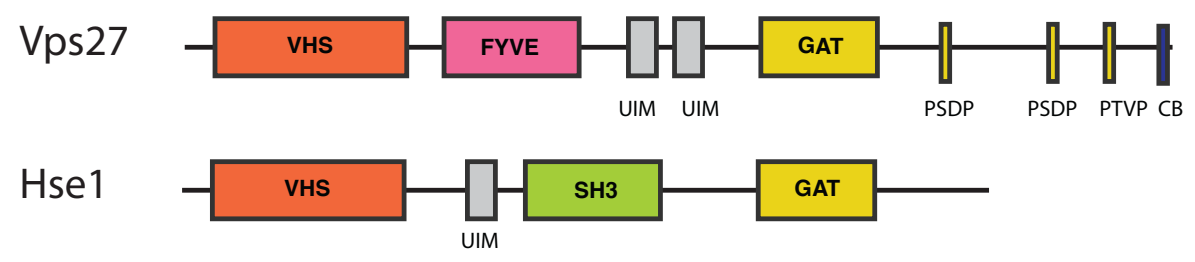

\section{Ubiquitin}

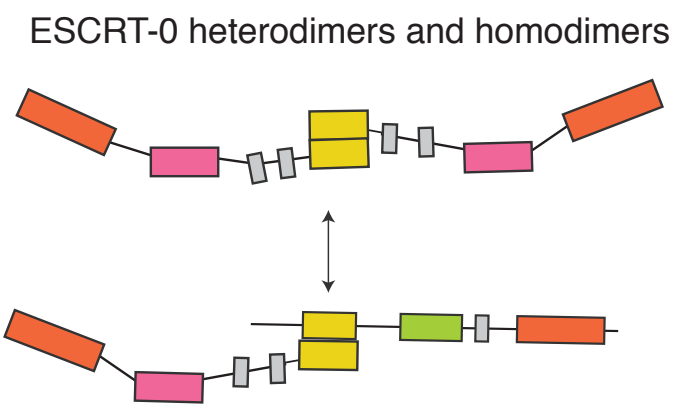

Weak self-assembly between ESCRT-0 heterodimers

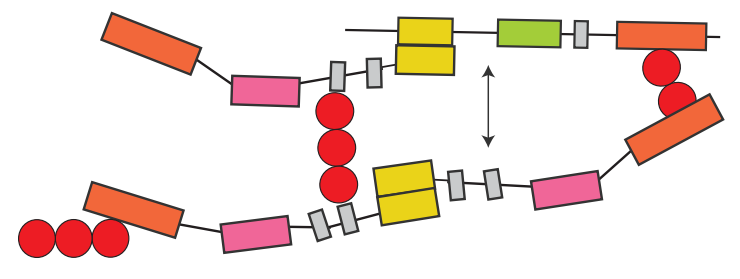

Enhanced self-assembly due to close proximity
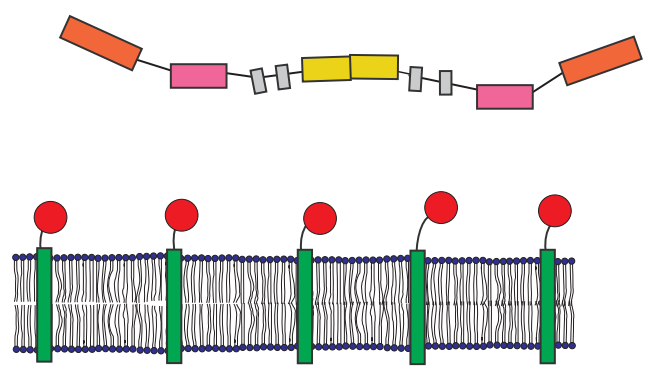

ESCRT-0 in solution, no self-association with no PI3P on membrane and mono-ubiquitinated cargo

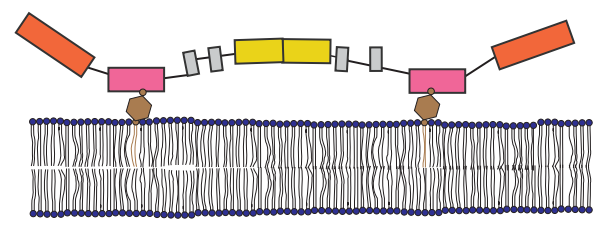

Higher density of PI3P enhance association rates of ESCRT-0 molecules, increasing propensity to self-assemble

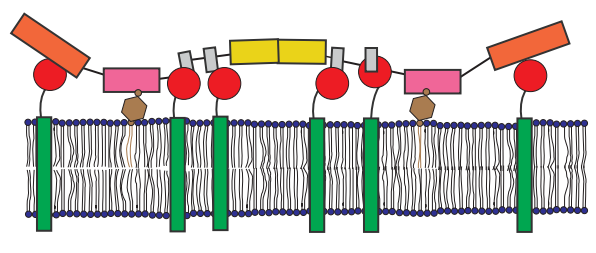

Recruitment and concentrated on 2D surface, leading to enhanced self association and cargo clustering 
S10. ESCRT-0 and ubiquitin assembly models on the surface of membranes. Surface density as regulated by lipid species, multivalency through polyubiquitin and multiple ubiquitin binding sites, and self-assembly through homo and hetero-oligomerization synergize to affect cargo condensation on membranes. 